\title{
Abolished miR158 activity leads to 21-nucleotide tertiary phasiRNA biogenesis that targets NHX2 in Arabidopsis thaliana
}

Abhinandan Mani Tripathi ${ }^{1,2,4}$, Rajneesh Singh ${ }^{1}$, Akanksha Singh ${ }^{1,2}$, Ashwani Kumar Verma ${ }^{1,2}$, Parneeta Mishra $^{1,2}$, Shiv Narayan ${ }^{3,2}$, Pramod Arvind Shirke ${ }^{3,2}$, Sribash Roy ${ }^{1,2} *$

\section{Affiliations:}

1. Molecular Biology and Biotechnology Division, CSIR-National Botanical Research Institute, Lucknow, 226001, India

2. Academy of Scientific and Innovative Research (AcSIR), Ghaziabad, 201002, India.

3. Plant Physiology Laboratory, CSIR-National Botanical Research Institute, Lucknow, 226001, India

4. Department of Ecology, Evolution and Behavior, Alexander Silberman Institute of Life Sciences, Faculty of Science, The Hebrew University of Jerusalem, Jerusalem, 9190401, Israel.

* Corresponding Author: Sribash Roy

Email: sribashroy@nbri.res.in

Short Title: Tertiary siRNA from miR158 target regulate $N H X 2$ 


\section{ABSTRACT}

Small RNAs including microRNAs (miRNAs) are short 20-24-nucleotide non-coding

3 RNAs. They are key regulators of gene expression in plants and other organisms. Some small

4 RNAs, mostly 22-nucleotide long trigger biogenesis of secondary small interfering RNAs

5 (siRNAs). Those siRNAs having distinctive phased configuration are known as phased siRNAs

6 (phasiRNAs) and act either in cis or trans enhancing silencing cascade. Here, we report natural

7 variants of MIR158 having deletions or insertions led to negligible or reduced expression of

8 miR158. The deletion/insertion events affected processing of primary transcript of miR158 to

9 precursor and to mature miR158. We show that miR158 targets a pseudo-pentatricopeptide gene

10 and its abolished activity led to 21-nucleotide tertiary phasiRNA generation from its target. The

11 biogenesis of these phasiRNAS is triggered by TAS2 derived two siRNAs. Accordingly, small

12 RNA analyses of these natural variants, mutants and over-expression lines of MIR158 or its

13 target exhibited enhanced or reduced phasiRNA biogenesis. Finally, we functionally validated

14 the highest expressed tertiary phasiRNA that targets $N H X 2$ thereby regulating transpiration and

15 stomatal conductance. Overall, we deciphered a new module of small RNA network, miRNA-

$16 T A S$-siRNA-pseudogene-tertiary phasiRNA-NHX2, suggesting an additional layer of gene

17 regulation and larger role of pseudogene in plants.

INTRODUCTION

Small RNAs (sRNAs) are typically 20 to 24 nucleotides long non-coding RNAs that act as important gene and chromatin regulators in plants. miRNAs are a class of small RNAs those negatively regulate gene expression either by homology mediated cleavage of mRNA or by

22 translational inhibition affecting many aspects of plant development (Bartel, 2004). They

23 originate from miRNA genes (MIRNA) transcribed by RNA polymerase II. The primary

24 transcripts (pri-miRNA) have characteristic stem-loop like secondary structures of various

25 lengths and complementary regions (Hirsch et al., 2006; Xie et al., 2005). The appropriate

26 secondary structures are then processed by RNase III enzyme Dicer-like1 (DCL1) (Bologna et

27 al., 2009; Zhang et al., 2017) forming pri-miRNA to precursor miRNA (pre-miRNA) and finally

28 to mature miRNA duplex (Voinnet, 2009). One strand of the mature miRNA duplex gets

29 incorporated into ARGONAUTE (AGO) protein to form a RNA-induced silencing complex 
30 (RISC) and carry out either post-transcriptional gene silencing or translational inhibition (Bartel,

31 2004).

32 Besides target degradation or translational inhibition, some miRNAs also act as a trigger

33 for biogenesis of another class of small RNAs, the tasiRNAs (trans acting small interfering 34 RNAs) or phasiRNAs (phased small interfering RNAs) to target downstream genes (Allen et al., 35 2005; Yoshikawa et al., 2005). The loci that generate such siRNAs are known as PHAS loci (Liu 36 et al., 2020). The triggering at the target PHAS locus can be mediated either by a single 22-nt 37 miRNA (one hit model) or by two 21-nt miRNAs (two hit model) (Axtell et al., 2006; Fei et al., 38 2013). After cleavage, the $3^{\prime}$ fragment is converted to dsRNA by RNA DEPENDENT RNA 39 POLYMERASE6 (RDR6) with the assistance of AGO1-RISC or AGO7-RISC and 40 SUPPRESSOR OF GENE SILENCING3 (SGS3). This dsRNA is then sequentially processed by 41 DCLs with the help of DOUBLE-STRANDED RNA BINDING FACTORS into 21- or 2442 nucleotides secondary siRNAs from the 5' end of the template strand (Adenot et al., 2006; 43 Vazquez et al., 2004). In two hit model, well studied in Arabidopsis TAS3, although two miR390 44 bind to the PHAS locus, but only the 3' proximal end is cleaved while 5' binding site defines the 45 boundary making tasiRNA production efficient, precise and accurate (Axtell et al., 2006; Liu et 46 al., 2020). However, in gymnosperms and eudicots the 5' miR390 target sites are cleavable likely 47 generating tasiRNAs in 5' to 3' direction (de Felippes et al., 2017). The bidirectional processing 48 mechanism has not been reported in Arabidopsis (Xia et al., 2017). However, using artificial 49 trans-acting siRNA transcript constructs the twin cleavage was reported in Arabidopsis but with 50 poor phasing of siRNAs (de Felippes et al., 2017).

PHAS loci can be located both within protein-coding and non-coding genes (Fei et al., 2013). Among the non-coding PHAS loci, the TAS loci, their targets and derived phasiRNA have 53 been well studied. In model plant Arabidopsis thaliana (A. thaliana), TAS1a, TAS1b, TAS1c and 54 TAS2 are targeted by miR173, and their 3' cleavage products produce either identical or very 55 closely related tasiRNAs (Yoshikawa et al., 2005). TAS3 and TAS4 are targeted by miR390 and 56 miR828, respectively and derived tasiRNA target three auxin response factors: ARF2, ARF3, and ARF4 (Allen et al., 2005; Williams et al., 2005) and MYB transcription factors (Xia et al., 2012). Non-coding PHAS loci have been reported from other plant species as well (Huang et al.,

59 2019; Xia et al., 2015). On the other hand, PHAS loci within protein-coding genes are leucine60 rich repeat $(N L R)$ genes (Zhai et al., 2011), MYB transcription factor (TF) genes (Xia et al., 
2013), AUXIN RESPONSE FACTOR (ARF) genes (Xia et al., 2017), NAC TF genes (SosaValencia et al., 2017) and PENTATRICOPEPTIDE REPEAT (PPR) genes (Chen et al., 2007; Howell et al., 2007) etc. Importantly, some $P P R$ also produces tertiary siRNAs whose production is triggered by tasiRNAs derived from TAS or TAS-like genes (Howell et al., 2007; Xia et al., 2013). This pathway of miRNA-TASL-PPR-siRNA was reported to be conserved in many plant species (Xia et al., 2013). Thus it is still unexplored if miRNA acts as a negative regulator of phasiRNA biogenesis. Moreover, though the biological functions of different phasiRNAs are well documented (Marin et al., 2010; Shahid et al., 2018; Zhou et al., 2013), the role of tertiary phasiRNAs is yet to be illuminated (Jia et al., 2020).

miR158 is amongst the first released 16 plant miRNAs (Reinhart et al., 2002). It is a nonconserved young miRNA, found only in members of Brassicaceae (Supplemental Figure 1A) (Fahlgren et al., 2007; Rajagopalan et al., 2006). Later it was identified in several cruciferous plants such as Arabidopsis lyrata (Fahlgren et al., 2010), Brassica oleracea (Wang et al., 2012), Raphanus sativus (Wang et al., 2015) and Brassica napus (Xu et al., 2012). In A. thaliana, it has two family member ath-miR158a and ath-miR158b (Bartel, 2004) (Supplemental Figure 1B). More recently it has been shown that miR158 play role in pollen development by regulating PPR in Brassica campestris (Ma et al., 2017).

A. thaliana harbors wide genetic variations among wild types and have been explored extensively over the decades (Alonso-Blanco and Koornneef, 2000; Mitchell-Olds and Schmitt, 2006). Effects of single nucleotide polymorphisms in the MIRNA have also been reported (Ryan et al., 2010; Todesco et al., 2012). In our previous study, we observed negligible expression of miR158 in a particular A. thaliana population of Indian west Himalayas under different growing conditions (Tripathi et al., 2019). These populations are reported from elevation range of $700 \mathrm{~m}$

84 above mean sea level (amsl) to $3400 \mathrm{~m}$ amsl having varied climatic conditions. Here, we show that negligible or moderate expression of miR158 is due to deletion and insertion events respectively, in MIR158 affecting it's processing to mature miRNA. miR158 targets a pseudo$P P R$. However, null expression of miR158 led to enhanced tertiary phasiRNAs biogenesis from pseudo- $P P R$ via TAS2 derived two siRNA triggers. Finally, we show that one of the tertiary phasiRNAs targets $N H X 2$ thereby regulating physiological processes prompting plants to complete life cycle early, presumably as a strategy of local adaptation. 


\section{RESULTS}

\section{$93 \quad$ Population specific expression of miR158}

94 Our previous analyses of small RNA profiling of the three populations Deh, Mun, and 95 Chit showed negligible expression of miR158 in Mun as compared to the other two populations, 96 both under field and controlled growth conditions (Table S4 of(Tripathi et al., 2019)). To 97 reconfirm the negligible expression of miR158 in Mun, we analyzed its expression under native, 98 controlled and common garden conditions along with miR158 SALK mutant using quantitative 99 real time PCR (qRT-PCR). The expression of miR158 was consistently negligible in Mun under 100 controlled, native and common garden conditions and as expected, in miR158 mutant (Figure1A; 101 Supplemental Figure 2A and 2B) as compared to the other two populations. We also checked the 102 relative expression pattern of miR158a, miR158b, and miR158a-3p arm and miR158a-5p arm 103 under controlled condition. Data suggested the relative expression of miR158a and miR158-3p 104 was significantly dominant over miR158b and miR158-5p respectively, in all the populations 105 (Supplemental Dataset 1; Supplemental Figure2C and 2D).

\section{Polymorphisms in MIRNA158 are unique to the west Himalayan populations}

To find out probable reason of population specific expression of miR158, we first analyzed the putative promoter sequence of miR158 (one kb upstream of transcription start site,

110 out the low expression of miR158 in Mun was due to differential promoter activity 111 (Supplemental Figure 3). However, PCR amplification and sequencing of MIRNA158 112 (MIRNA158a) from a few plants of each of the three populations exhibited length 113 polymorphisms (Figure 1B and 1C). Subsequently, 50 plants from each of the three populations 114 were analyzed (Supplemental Figure 4). The results indicated that while the 535 base pair (bp) 115 MIRNA158 of reference Col-0 (Szarzynska et al., 2009) (hereafter NTL, Normal Type Locus) 116 was present in all Chit plants, the Mun population harbors two different lengths of MIRNA158, a $117437 \mathrm{bp}$ locus having discontinuous deletion of total $98 \mathrm{bp}$ (starting at $10 \mathrm{bp}$ downstream of 118 transcription start site, TSS including four nucleotides of 5' precursor; hereafter DTL, Deleted 119 Type Locus) and a760 bp locus with an insertion of 225 bp (10 bp downstream of TSS; here after 120 ITL, Inserted Type Locus). Notably, the frequency of occurrence of DTL in Mun was 0.94, 121 remaining being ITL, and Deh harbors both NTL and ITL having frequency of 0.5 each (Figure 122 1B and 1D; Supplemental Figure 5). 
To examine if the insertion/deletion polymorphisms were present in the primary

124 transcript as well, we PCR amplified the locus using cDNA as template. Amplified product

125 exhibited similar length polymorphisms as observed with genomic DNA, ruling out the observed

126 polymorphisms was due to any splice variants (Supplemental Figure 6). We checked if these

127 polymorphisms are unique to the west Himalayan populations by comparing the MIRNA158

128 sequences from available 1001 genomes of A. thaliana (Alonso-Blanco et al., 2016). The

129 observed insertion/deletion polymorphisms are unique to the west Himalayan populations.

130 Further, the inserted fragment was not a transposable element (TE), as BLAST with TE database

131 (TAIR) did not retrieve any TE. However, the inserted fragment showed $97 \%$ similarity with a

132 sequence of chromosome four while MIRNA158 is located on chromosome three of A. thaliana.

133 Thus, the insertion event may be due to inter-chromosomal translocation.

\section{Polymorphisms in MIRNA158 affect its expression pattern}

135 We checked the expression pattern of miR158 in NTL, ITL and DTL by qRT-PCR. Data

136 suggested the expression of miR158 was the highest, intermediate, and negligible in NTL, ITL

137 and DTL, respectively (Figure 2A; Supplemental Dataset 2). In addition, we determined the

138 expression level of both pri- and pre-miR158. As expected, the level of pri-miR158 was more or

139 less similar in all the three variants (Figure 2B). However, pre-miR158 level was high,

140 intermediate, and low in NTL, ITL and DTL, respectively as observed with mature miR158

141 (Figure 2C). It further suggests that the variations in the expression of miR158 was not due to

142 promoter activity but presumably, may be due to differential processing of primary transcripts to

143 precursors. We evaluated the secondary structures of all the three different primary transcript

144 sequences from NTL, ITL and DTL plants. The secondary structure prediction of all the variants

145 revealed that the lower stem and their internal loops in DTL plants were structurally different

146 (disturbed) from the canonical ones and so was the large internal loop in ITL plants but these

147 were intact in NTL plants (Figure 2D - 2F). However, there were no such structural anomalies in

148 the predicted secondary structures of precursor sequences of all the three variants

149 (SupplementalFigure7). Any anomaly in the lower stem and internal loop structure can lead to

150 sloppy primary transcript processing (Mateos et al., 2010; Zhu et al., 2013). 
The targets of miR158 were predicted using psRNATarget (Dai and Zhao, 2011). Four transducing/WD40 repeat (BUB3.2) and pentatricopeptide repeat $(P P R)$ proteins were identified

157 as the putative targets of miR158 (Supplemental Dataset 3). We attempted to validate the 158 predicted targets by modified 5' RLM-RACE and it identified pseudo-PPR (AT1G62860.1) as 159 the only target of miR158 (20/20 clones). Quantification of the pseudo-PPR transcripts by qRT160 PCR also showed inverse expression pattern with miR158 (Figure 3A and 3B), thus confirming 161 it as the target of miR158. Further, the available degradome data (GSE55151) (Thatcher et al., 162 2015) analyses ( $\mathrm{P}$ value $<0.0001)$ also retrieved the identified pseudo- $P P R$ as the target of 163 miR158 (Supplemental Figure 8). Our repeated attempts failed to identify any of the other 164 predicted genes as the true target of miR158.

\section{The target pseudo-PPR acts as a PHAS locus in absence of miR158 activity}

Pseudogenes have been reported to act as a sponge for some miRNAs and thus protect the cognate target from degradation (Yu et al., 2014). However, our observation that miR158 cleaves the pseudogene prompted us for detail investigation. The target pseudo- $P P R$ besides harboring several stop codons also contains an open reading frame of 280 amino acid residues (Supplemental Figure 9). The parental PPR (AT1G64100.1) shares 78\% identity with the target pseudo-PPR. The PPRs are mostly known to involve in RNA editing (Yagi et al., 2013) and

172 some act as PHAS loci (Howell et al., 2007). PPRs are separated into two major classes based on 173 the nature of their PPR motifs, the P and PLS classes (Schmitz-Linneweber and Small, 2008; Xia 174 et al., 2013).Phylogenetic analysis of the target pseudo-PPR with 297 A. thaliana PPRs (Lurin et 175 al., 2004) suggested it is a P-type PPR (Supplemental Figure 10). Being a pseudogene, we ruled 176 out the possibility of its involvement in RNA editing and attempted to find if it acts as a PHAS 177 locus. PHAS loci are characterized by being targeted either by one 22-nucleotide small RNA 178 (one hit model) or by two 21-nucleotide small RNAs (two hit model) (Fei et al., 2013). We 179 analyzed the available degradome data to determine the putative small RNAs that might cleave 180 the pseudo- $P P R$. Our analysis indicated that apart from miR158, the pseudo- $P P R$ is also the 181 putative target of tasiRNAs, atTAS2 (2)-siR9(-) (hereafter after referred as siR9) and atTAS2(2)182 siR12(-) (hereafter after referred siR12) derived from TAS2 (Zhang et al., 2014) (Supplemental 
183 Figure 8). We validated the target cleavage by both the tasiRNAs using modified 5' RLM-RACE 184 and detected both the cleaved ends (Figure 3C and 3D).

Next, we mapped our small RNA libraries against the pseudo-PPR. A large number of pseudo-PPR mapped small RNAs (90\%) lied between siR9 and siR12 binding sites (Figure 4A).

187 Moreover, the small RNAs mapped between siR9 and siR12 were in phase with phasi score of 1881.38 to 35.0 ( $\mathrm{P}$ value < 0.0001) (Figure 4B) starting with the cleavage site of siR9 (702 nucleotides, from 5' end of pseudo-PPR) (Figure 4C). Although siR9 is reported to be 21nucleotide (Howell et al., 2007; Zhang et al., 2014; Zhong et al., 2013) but our small RNA analysis suggested higher expression of 22-nucleotide siR9 than the 21-nucleotide ones and siR12 were of 21-nucleotide (Figure 4E and Supplemental Table 1). Further, when small RNAs of each of the populations were mapped individually against the pseudo- $P P R$, higher number of small RNAs mapped between siR9 and siR12 binding sites in Mun than the other two populations (Supplemental Figure 11A). Additionally, out of 3138, a total of 830 non-redundant phasiRNAs from all the populations were identified those were derived from the pseudo- $P P R$ (here after referred as PDP) (Supplemental Dataset 4). Majority of the PDPs were 21-nucleotide while a very small fraction was of 22-nucleotide (Supplemental Figure11B). Further, around $45 \%$ of these PDPs had characteristic 5' terminal uridine (Supplemental Figure 11B). Since many $P P R$ s are known to act as PHAS loci and phasiRNAs produced from them may show redundancy due to repetitive domains, we speculated that the PDPs may be identical with the $P P R$ derived phasiRNAs. However, when we compared our pseudo- $P P R$ derived small RNAs with the small RNAs derived from each of the known 23 PPR PHAS loci (Howell et al., 2007), only 8\% of the PDPs showed similarity with other $P P R$ derived small RNAs (Supplemental Dataset 5) reconfirming pseudo-PPR acts as PHAS locus.

It was clearly evident that the abundance of PDPs was more in Mun than Deh and Chit (P 207 value $=0.005645, \mathrm{~F}=4.32423)$ (Table 1; Supplemental Figure 12A). Since above small RNA 208 libraries were prepared from pooled leaf samples of several plants of each population (Tripathi et 209 al., 2019), we re-examined the data using small RNA sequence data from randomly selected 210 individual plants of each variant types. Small RNA data from individual DTL, ITL and NTL 211 plant reconfirmed the above observations (Table 1; Figure 4D). Similarly, PDP analyses of 212 miR158 mutant, miR158 target pseudo-PPR mutant and Col-0 confirmed the higher expression 213 of PDPs in miR158 mutants and lower in pseudo-PPR mutant (Table 1; Supplemental Figure 
214 12B). Further over-expression lines of both miR158 (miR158 OE) and pseudo-PPR (pseudo-

$215 P P R$ OE) exhibited lower and higher expression of PDP, respectively as compared to Col-0

216 (Table 1; Supplemental Figure 12C). These analyses suggest miR158 negatively regulate the

217 PDP biogenesis.

218 The highly expressed PDP targets $\mathrm{NHX2}$

219 Most of the 21-nucleotide siRNAs are known to involve in AGO1 mediated cleavage of

220 target mRNA (Deng et al., 2018). In order to identify if any PDPs were associated with AGO

221 proteins, we took advantage of previously published AGO1 and AGO4 bound small RNA data

222 sets (GSE28591) (Wang et al., 2011). We identified a total of 200 PDPs identical with AGO1-

223 associated and 25 PDPs were identical with AGO4-associated small RNAs (Supplemental

224 Dataset 6). It presumably indicates a large number of the PDPs might involve in cleavage 225 mediated functions rather than involve in RdDM pathway. For further characterization of PDPs, 226 we focused on 11 PDPs which were derived from a significant cluster (Start: 746; End: 997; P 227 value $<0.00001, \mathrm{n}=11, \mathrm{k}=236$ ) (Supplemental Dataset 7) and were in phase with siR9 cleavage 228 site. To find out the cleaved ends of mRNAs by these PDPs, available degradome data was 229 analyzed. It identified three different mRNAs belonging to degradome category-2 (P value < 230 0.05), which indicates $>1$ raw read mapped at the position and abundance at position is less than 231 the maximum but higher than the median for the transcript (Supplemental Dataset 8). These three 232 mRNAs were putative target of one of the highly expressed PDP, PDP956-977 (+) 233 (Supplemental Dataset 8; Supplemental Figure 13). The best target identified was $N H X 2$, a $\mathrm{Na}^{+}-$

$234 \mathrm{~K}^{+} / \mathrm{H}^{+}$antiporter protein expressed in stomatal guard cell that maintain turgor to regulate 235 transpiration (Andrés et al., 2014; Barragán et al., 2012; Bassil et al., 2011). Other two targets 236 were identified as DNAJ heat shock N-terminal domain-containing proteins (AT2G05250.1 and 237 AT2G05230.1). Target validation using modified 5' RLM-RACE also identified NHX2 as the 238 target of PDP956-977 (+) (Figure 5A). Subsequently, we checked the expression of NHX2 in 239 DTL and miR158 mutant plants by qRT-PCR. As expected the expression of NHX2 in DTL and 240 miR158 mutant was lower than other lines (Figure 5B; Supplemental Figure 14A). Notably, the 241 expression of this gene in Mun was also found to be lower in our earlier global RNA sequencing 242 data (Tyagi et al., 2016).

$243 \quad N H X 1$ and $N H X 2$ are members of $N H X$ gene family and are involved in regulating 244 stomatal function (Barragán et al., 2012; Bassil et al., 2011). NHX2 shows a diurnal expression 
245 pattern and expression of $N H X 1$ increases in $N H X 2$ mutant to counter balance the NHX2 activity

246 (Andrés et al., 2014).We quantified the expression of NHX1 and NHX2 at different time points of

247 the day (Supplemental Figure 14B and 14C). In contrast to earlier observation, we did not

248 observe increased expression of NHX1 in miR158 mutants, as expected with NHX2 mutant. This

249 may be due to knock-out effect of $N H X 2$ as compared to knock-down effect in miR158 mutant.

250 Further, the maximum expression of $N H X 2$ was observed at $4 \mathrm{hr}$ of light in Col-0 and miR158

251 OE while in miR158 its expression was the maximum at $8 \mathrm{hr}$ of light (Supplemental Figure

252 14C). We speculated that the observed expression pattern of $N H X 2$ might be due to diurnal

253 variation in expression of either miR158 or its target pseudo-PPR. However, though there was no

254 variation in expression of miR158 at different time points of the day, the expression of pseudo-

$255 P P R$ was maximum at $8 \mathrm{hr}$ followed by at $4 \mathrm{hr}$ (Supplemental Figure 14D). Our results indicate

256 diurnal expression pattern of both pseudo-PPR and $N H X 2$ might be due to diurnal variation of

257 stoichiometric ratio between miR158 and pseudo-PPR.

\section{Down regulation of $\mathrm{NHX} 2$ by PDP affects physiological processes}

259 NHXs, mainly NHX1 and NHX2 are essential for turgor regulation and stomatal function

260 (Andrés et al., 2014; Barragán et al., 2012). Therefore, to examine if PDP956-977 (+) enriched

261 plants having altered NHX2 expression also exhibit impaired stomatal functioning, we measured

262 transpiration, stomatal conductance and water use efficiency of miR158 mutant, miR158 OE,

263 pseudo- $P P R$ mutant and pseudo- $P P R$ OE lines at different time points of the day. All the lines

264 showed reduced transpiration and stomatal conductance at different time points compared to the

265 control, except miR158 mutant, whose transpiration rate was slightly higher at 8 hr light

266 exposure and at dark (Figure 5C and 5D; Supplemental Dataset 9). Interestingly, pseudo-PPR

267 OE also exhibited lower rate of transpiration and stomatal conductance as compared to the

268 control, presumably due to higher stoichiometric ratio in favor of pseudo- $P P R$ over miR158.

269 Further, transpiration rate and stomatal conductance were maximum at $4 \mathrm{hr}$ light and gradually

270 decreased after $8 \mathrm{hr}$ of light exposure in all but miR158 mutant and pseudo-PPR OE and finally

271 all reached the lowest level at dark (after 2 hr 30 min dark). The impaired stomatal activity in

272 miR158 mutant and miR158 OE was further examined by imaging the stomatal aperture at light

273 and dark under scanning electron-microscope. It is evident that stomatal closure was delayed in

274 miR158 mutant under dark as compared to control (Figure 5E; Supplemental Figure 15A). Also,

275 miR158 mutant exhibited significantly higher water use efficiency (Figure 5F) and lower rate of 
water loss after $2 \mathrm{hr} 45 \mathrm{~min}$ as compared to the control plants (Supplemental Figure 15B;

277 Supplemental Dataset 9). Accordingly, when subjected to water-holding for 35 days, the miR158

278 mutant and pseudo-PPR OE could complete their life cycle, while the growth of the control Col-

2790 , miR158 OE and pseudo-PPR mutant were severely affected and could not complete their life

280 cycle (Figure 5G). Similar observations were recorded with NTL, ITL and DTL plants

281 (Supplemental Figure 15C-15H). These results indicate biogenesis of PDP driven by miR 158

282 regulate transpiration and stomatal conductance via $N H X 2$.

283 Finally, we evaluated the effect of these altered physiological processes on plants growth

284 and development by recording various life cycle and morphological parameters (Figure 5H;

285 Supplemental Figure 16, Supplemental Dataset 9). Data suggested the miR158 mutant possessed

286 elongated leaves and petioles with larger rosette area (Supplemental Figure 17) and bolted six

287 days ( \pm 1 day) earlier as compared to the Col-0 (Figure $5 \mathrm{H})$. Bolting was delayed by 21 days $( \pm 2$

288 days) in over expression lines than Col-0. Similarly, pseudo- $P P R$ mutant matured 11 days $( \pm 1$

289 days) earlier than it's over expression lines (Figure 5H and Supplemental Figure 16). Overall,

290 both physiological and morphological data indicate miR158 regulate growth and development of

291 A. thaliana by regulating NHX2 via PDPs.

\section{DISCUSSION}

\section{Natural variations in MIR158 affect pri-miR158 processing}

We identified natural variants of MIR158 in west Himalayan populations of A. thaliana.

295 The insertion/deletion events are unique to these populations, not observed in any other world

296 accessions. Though both insertion and deletions were observed in these populations, notable is

297 the discontinuous deletion one with very high frequency of occurrence (0.94) in a particular

298 population (Mun). Subsequently, we identified some other populations besides Deh, Mun and

299 Chit in the region and PCR screened the MIR158 in these new populations as well. Interestingly,

300 we could not identify any NTL plants in any of the populations including newly screened ones

301 except, Chit and Deh. Noteworthy to mention here that all these populations are from same

302 geographical continuity along the Himalayan elevation (700 m amsl to $2000 \mathrm{~m}$ amsl), except

303 Chit which is at the extreme elevation of $\sim 3400 \mathrm{~m}$ amsl and lies hundreds of kilometer apart. It

304 remains to be seen if abolition of miR158 activity in Mun population is under purifying selection

305 aiding in local adaptation. Intuitively so, as there is gradual decrease in frequency of NTL plants 
as one moves from lower elevation (Deh), harboring almost equal frequency of NTL and ITL to higher elevation (Mun)that harbors high frequency of DTL plants (0.94) with no NTL plants . affecting their processing efficiency and/or accuracy by plant miRNA processing machineries,

310 such as DCL1, HYL1, or SERRATE (Kurihara and Watanabe, 2004; Reinhart et al., 2002).

311 Particularly, the positioning of the initial DCL1 processing event in many precursors is

312 dependent on a lower stem of $\sim 15$ bp below the miRNA/miRNA* duplex followed by a large 313 internal loop (Cuperus et al., 2010; Mateos et al., 2010; Werner et al., 2010). These critical 314 features of secondary structures, particularly the lower stem structure and internal loop is 315 severely affected in DTL while in ITL, the internal loop is absent (Figure 2E and 2F). Thus the 316 miRNA processing machineries completely or partially failed to recognize the inappropriate 317 secondary structures (Figure 2E and 2F) resulting negligible or moderate expression in DTL and 318 ITL, respectively(Figure 2A). Earlier, 139 bp insertion in primary transcript of miR163 in $A$. 319 arenosa was shown to affect its processing leading to reduced expression (Ng et al., 2011). 320 Different miRNAs may be processed by different mechanisms depending on the sequential 321 direction of the miRNA processing machineries. miR158 is reported to be processed following 322 canonical base-to-loop mechanism, where the first cut is made by counting a 15-nt stem region 323 above the internal loop (Bologna et al., 2013). Overall, the insertion/deletion make secondary 324 structures of pri-miR158 in appropriate for plant RNA processing machineries leading to reduced 325 or negligible expression of miR158.

326 Abolished miR158 activity triggers PDP biogenesis following "two-hit, two-cleavage" model

Earlier it was reported that miR158 targets a $P P R$ and thereby regulates pollen development in Brassica campestris (Ma et al., 2017). However, our repeated attempts failed to identify the PPR as the target of miR158 in A. thaliana. Subsequently, based on our in-silico

331 target prediction results, we validate a pseudo- $P P R$ as its target. Though many pseudogenes are 332 reported to generate siRNAs both in plants and animals (Guo et al., 2009; Thibaud-Nissen et al., 333 2009) and also act as sponge for miRNA (An et al., 2017; Yu et al., 2014), our findings that 334 miR158 targets and cleaves a pseudogene may widen the range of biological functions of plant 335 pseudogenes. Indeed, in absence of miR158 activity, the TAS2 derived siRNAs, siR9 and siR12 336 bind to miR158 target pseudo-PPR and subsequently processed to phasiRNAs (Table 1). Thus, a 
337 large number of PDPs were generated in DTL and MIR158 mutant plants as compared to NTL 338 and ITL. Conversely, we detected negligible PDPs in pseudo-PPR mutant and miR158 OE lines. 339 Although there is report that TAS2-derived siRNAs act as a trigger of tasiRNAs (e.g., 340 tasiRNA2140) biogenesis from PPR, there is scanty of report showing cleavage of target by both 341 the tasiRNAs for biogenesis of tertiary phasiRNAs (Chen et al., 2007; Howell et al., 2007).

342 Moreover, considering the one-hit model is the most prevalent pathway for phasiRNA 343 production and the two-hit model primarily reported from TAS3, the observed pathway of 344 phasiRNA production implicates diversity in their biogenesis. More importantly, we observed 345 phasiRNA production from both 5' and 3' cleaved ends of the target, albeit in higher number 346 from 5' cleaved end than 3' cleaved end. Recently, similar two end target cleavage and 347 phasiRNAs production from downstream of both cleavage sites were reported in wild strawberry 348 (Fragaria vesca) TAS3S (Xia et al., 2015). The above study together with present report suggest 349 in addition to prevalent "two-hit, one-cleavage" configuration for tasiRNA production (Liu et al., 350 2020), a "two-hit, two-cleavage' configuration may be operating as well, at least in some plant 351 species.

\section{A new module of phasiRNA regulation}

353 Our observation that pseudogene act as a template for phasiRNA biogenesis implicates 354 larger role of pseudogenes in plants. Firstly, pseudogenes are known template for 24- nucleotide 355 siRNA biogenesis, primarily involve in RNA directed DNA methylation (Guo et al., 2009). In 356 contrast, we observed a large number of 21- nucleotide PDPs those were associated with AGO1

357 (Supplemental Figure 11b and Supplemental Data 6) indicating probable involvement in 358 cleavage mediated function. Secondly, pseudogene derived siRNA targeting of protein coding 359 gene is not known in plants. Here, we show that one of the PDPs targets $N H X 2$, a member of 360 NHX gene family involve in vacuolar $\mathrm{K}^{+}$homeostasis (Barragán et al., 2012). Further, in other 361 organisms most of the pseudogene derived siRNAs are known to target their parent protein 362 coding genes (Tam et al., 2008; Watanabe et al., 2008) rather than non-parental gene. The PDPs 363 neither showed significant similarity with parent $P P R$ (AT1G64100) nor any $P P R$ target could 364 be identified in degradome data. The targeting of $N H X 2$ by one of the PDPs also contradicts 365 earlier report that opined against secondary targeting by clad-derived siRNAs on non-clad target 366 transcripts, especially in case of $P P R$ derived phasiRNAs (Howell et al., 2007). Noteworthy to 367 mention that phasiRNAs production is restricted only to P-type $P P R$ in plants (Howell et al., 
2007; Xia et al., 2013) and phylogenetic analysis of the template pseudo- $P P R$ shows it belongs to P-type PPR clad (Supplemental Figure10). Secondary siRNA biogenesis pathway involving PPRs following the miRNA-TAS like gene-PPR-siRNA module (Xia et al., 2013) or the most common pathway i.e miRNA-TAS-siRNA are mainly triggered by 22-nucleotide siRNAs ( a few

372 are of 21- nucleotide e.g., miR156 and miR172) following "two hit, one cleavage" model (Zhai

373 et al., 2011). We demonstrate another layer of 21- nucleotide phasiRNA biogenesis, miRNA-

$374 T A S$-siRNA-pseudo-PPR-tertiary phasiRNA-NHX2 that regulates a protein coding gene of far

375 distant family following "two hit, two cleavage" model (Figure 6).

PDP mediated down-regulation of $\mathrm{NHX} 2$ affects physiological processes in $A$. thaliana

The down-regulation of NHX2 by a PDP (Figure 5B) led to reduced transpiration, stomatal conductance and better water use efficiency (Figure 5C-5G). The reduced transpiration and stomatal conductance in miR158 mutant and pseudo-PPR OE as compared to control is in accordance with earlier observation of NHX2 mediated regulation of these activities (Andrés et al., 2014). The delayed response of stomatal functioning has been attributed to NHX proteins which help to maintain turgor by regulating $\mathrm{K}^{+}$homeostasis and it corroborates with the observed diurnal variations in NHX2 expression in different lines (Supplemental Figure 14D). Both opening and closure of stomata was reported to be affected in double knock out mutant of NHX1 and NHX2 in A. thaliana and the single mutant was more affected mainly in stomatal opening than closure (Andrés et al., 2014). It is evident that the stomatal opening was more impaired than closure in miR158 mutant as compared to other lines, as the rates of transpiration and stomatal conductance did not fall rapidly as others as dusk approached. In fact response to stomatal closure was delayed showing higher rates at dusk ( $8 \mathrm{hr})$ and even at dark ( $2 \mathrm{hr} 30 \mathrm{~min})$. The reduced transpiration could not be attributed to the defective guard cells (Figure 5E). Notably, miR158 mutant and to a lesser extent pseudo-PPR OE lines completed life cycle earlier than control both under water stress as well as under regular water supply conditions (Figure 5G and

$3935 \mathrm{H})$. The overall performance of pseudo-PPROE is in line with the observed phasiRNA 394 generation and probable stoichiomeric ratio between pseudo- $P P R$ and miR158 in favor of 395 pseudo- $P P R$ in this line. Thus the negligible expression of miR158 and enhanced phasiRNA 396 generation might be such a strategy of the population thriving under elevated area to complete 397 life cycle early. 
Natural variants of MIR158 led us to identify a module of 21- nucleotide phasiRNA biogenesis. The identified pathway widens the scope of phasiRNA biogenesis beyond prevalent pathways. miRNA driven cleavage of a pseudogene or regulation of a protein coding gene by a pseudogene derived phasiRNA highlights the significance of pseudogene as well as role of

402 tertiary phasiRNA in plants. However, although siR9 and siR12 act as a trigger for biogenesis of 403 phasiRNA, it will be interesting to examine if a 21- or 22- nucleotide miR158 can act as a trigger 404 for observed phasiRNA biogenesis from the cleaved end instead of template degradation.. 405 Understanding the evolutionary significance of such a pathway may shed light on siRNA 406 regulated local adaptation.

\section{METHODS}

\section{Plants materials and growth conditions}

The seeds of different populations of A. thaliana of west Himalayas (Deh, Mun, Chit), and SALK mutants lines (miR158 mutant, SALK_025691; pseudo-PPR muatnt, SALK_043644)

411 and Col-0 were grown under controlled condition of $22^{\circ} \mathrm{C}$ temperature, $140 \mu \mathrm{mol} / \mathrm{m}^{2} / \mathrm{sec}$ light 412 intensity and 16/8-hour day/night cycle. Besides, seeds were also grown in common garden at 413 CSIR-NBRI, Lucknow, Uttar Pradesh, India during the month of November to February to check 414 the influence of environment on the expression of miR158. The homozygous lines of SALK 415 mutants were selected as discribed in GABI (http://signal.salk.edu/tdnaprimers.2.html) using 416 primers listed in Supplemental Dataset 10.

\section{RNA isolation, small RNA sequencing and analysis}

418 Total RNA was isolated from rosette leaves of 28 days old plants using mirVana ${ }^{\mathrm{TM}}$ 419 miRNA isolation kit (Ambion, USA) following the manufacturer instructions. The quality of 420 RNA was checked on both agarose gel as well as using bioanalyzer (Agilent, USA). RNA 421 samples having RIN >7 were used for small RNA library preparation. Four pooled small RNA 422 libraries corresponding to Deh, Mun, Chit and Col-0 was prepared and used as replicates of 423 earlier sequenced data from the same populations (Tripathi et al., 2019). Further, for each plant 424 type individual libraries were prepared and sequenced in replicates, except for DTL, miR158 OE 425 and pseudo-PPR OE (one of these libraries failed the quality control).Libraries were prepared 426 using NEB Next Small RNA Library Prep Set (New England Biolabs) and sequencing was 427 carried out using Illumina HiSeq 4000 in single-end mode by outsourcing. Thus, a total of 20 
428 small RNA sequencing libraries data were analyzed (Supplemental Dataset 11).The raw 429 sequences were analyzed using UEA small RNA workbench (Version 4.5) (Stocks et al., 430 2018). Briefly, the adapters were removed from raw reads and size-filtered between 18-28 431 nucleotides using adapter removal tool. The filtered reads were mapped onto the reference 432 Arabidopsis TAIR10 genome using Bowtie2 with default parameters. The genome mapped reads 433 were used for identification and quantification of miRNA and tasiRNA using miRProf 434 implemented in UEA workbench with miRBase22 (http://www.mirbase.org/) and tasiRNA data 435 base (http://bioinfo.jit.edu.cn/tasiRNADatabase) (Zhang et al., 2014), as background, 436 respectively.

\section{PhasiRNA identification and scoring}

$438 \quad$ For identification of pseudo-PPR derived phasiRNA (PDP), the filtered small RNA reads 439 were mapped onto the pseudo-PPR reference sequence using Bowtie2.0 (zero mismatch) 440 (Langmead and Salzberg, 2012). It is worthy to mention that binding site of one of the TAS2 441 derived small RNA, siR9 on the pseudo- $P P R$ and its production site on TAS2 show perfect 442 complementary to each other (Supplemental Figure 17A). Therefore, in order to exclude any 443 ambiguous mapping of siR9 (higher expression as compared to PDPs) on pseudo- $P P R$, we 444 masked (replaced by N) half complementary sites of pseudo-PPR (Supplemental Figure 17B). 445 This led us to specifically identify the PDPs.

446 To identify whether this pseudo- $P P R$ acts as true phasi generating loci, the $\mathrm{p}$ value was 447 calculated using the tasi- predictor tool in-built in UEA workbench and pseudo- $P P R$ sequence as 448 reference following (Chen et al., 2007). Further, the phasing score was also calculated using 21449 nucleotide phasing register in a 10 cycle window and $\mathrm{p}>0.001$. Phasing score was calculated 450 following (De Paoli et al., 2009).

$$
\text { Phasing score }=\ln \left[\left(1+10 \times \frac{\sum_{\mathrm{i}=1}^{10} \mathrm{Pi}}{1+\sum \mathrm{U}}\right)^{\mathrm{n}-2}\right]
$$

451 where $\mathrm{n}$ is the number of phase registers occupied by at least one unique 21-nucleotide sRNA 452 within a ten-phase register window, $\mathrm{P}$ is the total number of reads for all 21-nucleotide sRNA 453 reads falling into a given phase in a given window, and $U$ is the total number of unique reads for 454 all 21-nucleotide sRNAs falling out of a given phase. 


\section{Promoter analysis and identification of polymorphism in MIR158}

The putative promoter sequences of MIR 158 (one kb upstream of transcription start sites) were extracted from our genome sequence data of Deh, Mun, and Chit and were checked for any sequence variations using multiple sequence alignment tool implemented in MEGA 6.0 (Tamura et al., 2013). During initial PCR amplification of MIR158 locus from a few plants of the three

460 populations, we detected length polymorphisms as visualized on $1.2 \%$ agarose gel which was 461 confirmed by sequencing of the amplified products. Subsequently, MIR158 locus was PCR 462 amplified and sequenced from 50 plants of each of the three populations using primers listed in 463 Supplementary Data 10. Multiple sequence alignment analysis was performed using MEGA 6.0. 464 To further confirm the length polymorphisms, we amplified the pri-miR158 locus using cDNA 465 as template from different populations using the length polymorphisms specific primers (NTL, 466 ITL and DTL specific primers, Supplementary Data 10). The miR158 locus of these populations 467 were compared with 1135 A. thaliana accessions as well using POLYMORPH 1001 tools 468 (https://tools.1001genomes.org/polymorph/) selecting nucleotides position range 336598246933666000 on chromosome 3 (range of miR158a locus).

Secondary structure prediction of pri-miR158

The primary transcript of miR158 amplified from each of the three populations were 472 sequenced and secondary structure of these primary transcripts were predicted using RNAfold 473 web server (http://rna.tbi.univie.ac.at/cgi-bin/RNAWebSuite/RNAfold.cgi) at default parameters.

\section{Target prediction and degradome analysis}

475 Targets of miRNA, tasiRNA and phasiRNA were predicted by psRNA target (Dai and 476 Zhao, 2011) using expectation value cutoff of less than three. Available degradome data of $A$. 477 thaliana (GSE55151) (Thatcher et al., 2015) was downloaded and analyzed using PAREsnip 478 (Folkes et al., 2012) following stringent criteria. Briefly, mismatch on position 10 and 11 were 479 not allowed, maximal numbers of mismatches were set to four and two mismatches on adjacent 480 positions were not allowed. The targets with lower confidence (category four and three) were not 481 considered. The T-plots were generated by VisSR tool (Stocks et al., 2018). 
484 carried out using GeneRacer ${ }^{\mathrm{TM}}$ Kit (Ambion, USA) following user's manual. Briefly, RNA was 485 isolated using RNeasy Mini Kit (Qiagen, USA). Adapter was ligated to $4 \mu \mathrm{g}$ freshly isolated 486 RNA at $37^{0} \mathrm{C}$ for one hours followed by $16{ }^{0} \mathrm{C}$ for eight hours. cDNA was synthesized using 487 random primers with ligated RNA. Touchdown PCR amplification was performed using the 488 adapter specific GeneRacer 5' primer and gene specific outer primers following PCR program of $4892 \mathrm{~min}$ at $94^{\circ} \mathrm{C}$, followed by five cycles of $30 \mathrm{~s}$ at $94^{\circ} \mathrm{C}$ and $90 \mathrm{~s}$ at $72^{\circ} \mathrm{C}$, five cycles of $30 \mathrm{~s}$ at $94^{\circ} \mathrm{C}$ 490 and $120 \mathrm{~s}$ at $70^{\circ} \mathrm{C}$, and 25 cycles of $30 \mathrm{~s}$ at $94^{\circ} \mathrm{C}, 30 \mathrm{~s}$ at $65^{\circ} \mathrm{C}$ and $90 \mathrm{~s}$ at $72^{\circ} \mathrm{C}$ and finally $10 \mathrm{~min}$ 491 at $72^{\circ} \mathrm{C}$. Nested PCR amplification was performed using the adapter specific GeneRacer 5' 492 Nested primer and gene specific inner primers and two microliter template from the initial 493 touchdown PCR following the PCR program of 2 min at $94^{\circ} \mathrm{C}$, followed by 25 cycles of 30 s at $49494^{\circ} \mathrm{C}, 30 \mathrm{~s}$ at $65^{\circ} \mathrm{C}$ and $2 \mathrm{~min}$ at $72^{\circ} \mathrm{C}$ and finally $10 \mathrm{~min}$ at $72^{\circ} \mathrm{C}$. The PCR product was run on $4952 \%$ agarose gel and the expected bands were eluted using Wizard ${ }^{\circledR}$ SV Gel and PCR cleanup kit 496 (Promega, USA),cloned in pGEM-T easy vector (Promega, USA), and at least five individual 497 clones were sequenced for each target.

\section{Expression analysis by qRT-PCR}

Expression level of miRNA158 primary transcripts, precursor and target genes were carried out using qRT-PCR. cDNAs were prepared by GoScript ${ }^{\mathrm{TM}}$ Reverse Transcription System

501 following manufactuere's protocol (Promega, USA). All qRT-PCRs were performed in ABI 7300 502 real-time PCR machine (Applied Biosystem, USA) using DyNAmo Flash SYBR Green 503 (Thermo, USA) with cycling conditions: denaturation at $95^{\circ} \mathrm{C}$ for $10 \mathrm{~min}$ followed by 40 cycles 504 of denaturation at $95^{\circ} \mathrm{C}$ for $20 \mathrm{~s}$, annealing and extension together at $60^{\circ} \mathrm{C}$ for $60 \mathrm{~s}$. The respective 505 primers are listed in Supplemental Dataset 10. All reactions were performed with three to five 506 biological and two technical replicates. 5s-rRNA and actin gene were used as endogenous 507 control for stem loop-PCR and target genes, respectively (Czechowski et al., 2005; May et al., 508 2013). The expression value of each sample was normalized using expression value of respective 509 endogenous control and relative expression was calculated. The fold change values were 510 determined using $2^{-\Delta \Delta C t}$ method (Schmittgen and Livak, 2008). Before conducting stem loop 511 qRT-PCR, the specificity of miR158 stem loop primers was checked using the end point PCR 512 following (Varkonyi-Gasic et al., 2007). Briefly, $100 \mathrm{ng}$ of total RNA was revesre transcribed 513 using miR158 stem loop primers and SuperScript ${ }^{\circledR}$ III Reverse Transcriptase (Invitrogen, USA) 514 following manufacturer's instructions. The polymerase chain reaction was performed using two 
$515 \mu 1$ of cDNA as template and miR158 specific forward primer, and stem-loop specific reverse 516 primer at thermocycler condition of $94^{\circ} \mathrm{C}$ for $2 \mathrm{~min}$, followed by 35 cycles of $94^{\circ} \mathrm{C}$ for $15 \mathrm{~s}$ and

$51760^{\circ} \mathrm{C}$ for $1 \mathrm{~min}$. The PCR product was checked on $2 \%$ agarose gel (Supplemental Figure 18).

518 The variation in expression of the primary transcripts of miR158a and miR158b was

519 taken as proxy to distinguish between the expression of miR158a and miR158b. This is because

520 the precursor sequences of miR158a and miR158b do not harbour sufficient variations

521 (Supplementary Fig.1b).

\section{Phylogenetic analysis of PPRs}

523297 PPRs having conserved PPR-domains amongst 450 reported A. thaliana PPRs(Lurin

524 et al., 2004) were considered for phylogenetic analysis. The sequences of the PPRs were 525 downloaded from TAIR (www.arabidopsis.org) and aligned using CLUSTAL-W implemented in 526 MEGA 6.0 (Tamura et al., 2013). Phylogenetic tree was constructed using Neighbor-Joining 527 method implemented in MEGA 6.0 with 1000 bootstrap replicates.

\section{Cloning and generation of over expression transgenic plants}

Pri-miR158 and pseudo-PPR fragments were PCR amplified using respective gene

530 specific primers (Supplemental Dataset 10) following standard PCR protocol. The amplified 531 fragments were cloned in pGEM-T cloning vector (Promega, USA) and subsequently transferred 532 into pBI121 binary vector. A. thaliana plants (Col-0 background) were transformed by floral dip 533 method (Clough and Bent, 1998). Transformants were screened on one-half-strength MS agar 534 plates containing kanamycin $(50 \mu \mathrm{g} / \mathrm{mL})$ followed by PCR screening. Subsequently, T3 535 homozygous plants were selected for further analyses. In spite of repeated attempts we were 536 unable to generate the complementation line of miR158 in DTL plants due to difficulty in getting 537 viable seeds after transformation due to unknown reasons.

\section{Measurement of morphological and physiological parameters}

539 Various morphological and life cycle parameters of ten five-weeks post-germinated

540 plants each of miR158 mutant, pseudo- $P P R$ mutant, transgenic lines and Col-0 were measured.

541 Leaf area was estimated as product of length and width. Leaf shape was represented as a ratio of

542 length to width. Rosette area was measured as product of major and minor axis and rosette shape 543 as the ratio of major to minor axis. Both leaf and rosette shape were considered as rounded when 544 the ratio is close to one and elongated if the ratio was more than one. Life cycle namely, bolting, 
545 first flower opening, emergence of first silique and senescence were measured in terms of days

546 after germination. Plants were monitored regularly and data were recorded at every two alternate

547 days.

548 Stomatal conductance, rate of transpiration, and water use efficiency of plants at bolting stage

549 (principal growth phase 5.10 (Boyes et al., 2001))were measured using LICOR-6400 portable

550 photosynthesis system (LI-COR, USA), at four different time points: Immediately after onset of

551 light, four and eight hours post exposure of light and two and half hours post exposure of dark.

552 Stomatal aperture was measured by peeling the epidermal layer of leaf lower surface and 553 mounted in glycerol and visualized under light microscope at 100X using LEICA DM2500.

554 Stomatal measurement was taken at two time points of four hours post light and two and half

555 hours of post dark exposure. Additionally, the stomata from these two time points were also 556 visualized and measured using scanning electron microscopy (FEI Quanta 250, Bionand, Spain).

557 The leaf tissues were fixed and processed according to (Bomblies et al., 2008). Briefly, the tissue 558 was fixed overnight at $4^{0} \mathrm{C}$ in FAA fixative (3.7\% formaldehyde, $50 \%$ ethanol, $5 \%$ acetic acid) 559 and then dehydrated serially in the ethanol solutions of $30 \%, 50 \%, 70 \%, 90 \%, 95 \%$ and $100 \%$

560 for about 1 hour each. The tissue was kept at $4^{0} \mathrm{C}$ overnight in $100 \%$ ethanol and then processed 561 for CPD (Critical-Point Drying) followed by gold coating and visualization at 8000x.

\section{Drought Assay}

563 Ten plants of each of miR158 mutant, miR158 OE, pseudo- $P P R$ mutant, pseudo- $P P R$ OE and

564 Col0 along with NTL, ITL and DTL plants were grown under controlled condition for three 565 weeks with normal watering. On the last day of third week pots were saturated with water and 566 soil surface was covered with plastic wrap. The plants were allowed to grow without additional 567 water and monitored till they survived. The photographs were taken after 35 days of water 568 holding.

\section{Relative Water Content}

570 The relative water content and water loss was measured following (Barrs and 571 Weatherley, 1962). Briefly, rosette leaves were harvested and soaked in water for 30 min to 572 make them completely turgid. The turgid weight of each leaf was recorded and then kept at room 573 temperature for varying intervals of time. The weight of each leaf was measured after $5 \mathrm{~min}$, $57415 \mathrm{~min}, 30 \mathrm{~min}, 1$ hour, 1.45 hour and 2.45 hour. After 2.45 hour, the leaves were kept at $65^{\circ} \mathrm{C}$ in 
575 dry oven for 72 hours and then measured the dry weight of the leaves. The relative water content 576 was measured using the formula:

$$
\text { RWC }(\%)=[(F W-D W) /(T W-D W)] \times 100,
$$

578 Where, $\mathrm{RWC}=$ relative water content, $\mathrm{FW}=$ fresh weight at different time points, $\mathrm{DW}=$ dry

579 weight, and TW=turgid weight

580 All the morphological and physiological data was analyzed using one-way ANOVA in R.

581 The multiple comparison analysis of the linear ANOVA model was conducted using 'glht'

582 function in multcomp package in $\mathrm{R}$.

\section{Supplemental Data}

584 Supplemental Figure 1. Sequence conservation of miR158 (Supports Figure 1)

585 Supplemental Figure 2. Relative Expression of miR158 in different populations as estimated by 586 stem-loop RT-PCR (Supports Figure 1)

587 Supplemental Figure 3. Multiple sequence alignment of miR158 promoter sequences from 588 different populations using CLUSRTAL-W (Supports Figure 1)

589 Supplemental Figure 4. Representative image of PCR amplification of miR158 locus showing 590 length polymorphisms in different populations of Indian west Himalayas (Supports Figure 1)

591 Supplemental Figure 5. Length polymorphism of miR158 loci in different populations 592 (Supports Figure 1)

593 Supplemental Figure 6. Length polymorphism in pri-miR158 locus in NTL, DTL and ITL 594 plants (Supports Figure 1)

595 Supplemental Figure 7. Predicted secondary structures of miR158 precursor (Supports Figure 596 2)

597 Supplemental Figure 8. Target plot (t-plot) for miR158 and tasiRNA targets identified using 598 degradome data (Supports Figure 3)

599 Supplemental Figure 9. Translated protein sequence of pseudo- $P P R$ with predicted ORFs (open 600 reading frames) (Supports Figure 3)

601 Supplemental Figure 10. Phylogenetic analysis of $P P R$ genes (Supports Figure 3)

602 Supplemental Figure 11. Abundance of phasiRNA (PDPs) in different populations (Supports 603 Figure 4) 
604 Supplemental Figure 12. Differential expression of PDPs using log2 values of read counts from 605 different plant samples (Supports Figure 4)

606 Supplemental Figure 13. Target-plot (T-plot) of NHX2 (Supports Figure 5)

607 Supplemental Figure 14. Relative expression pattern of $N H X 2, N H X 1$, pseudo- $P P R$ and 608 miR158 using qRT-PCR and stem-loop qRT-PCR (Supports Figure 6)

609 Supplemental Figure 15. Physiological parameters of different samples (Supports Figure 6)

610 Supplemental Figure 16. Growth and leaf size of different transgenics and Himalayan 611 populations (Supports Figure 6)

612 Supplemental Figure 17. Morphological variations in different transgenic lines and Col0 613 (Supports Figure 6)

614 Supplemental Figure 18. Masked sequence of pseudo- $P P R$ used for identifying pseudo- $P P R$ 615 derived phasiRNAs (PDPs) (Supports Figure 4)

616 Supplemental Figure 19. Gel Image of Endpoint PCR in the three populations (Supports Figure 617 1)

618 Supplemental Table 1. Length distribution of expressed form of siR9 and siR12 in NTL, ITL 619 and DTL.

620 Supplemental Dataset 1. Expression (read counts) of miRNAs, siR9 and siR12in different 621 populations of Indian west Himalayas

622 Supplemental Dataset 2. Expression (read counts) of miRNAs, siR9 and siR12 in NTL, ITL 623 and DTL.

624 Supplemental Dataset 3. List of putative targets of miR158 as predicted using psRNATarget.

625 Supplemental Dataset 4. Pseudo-PPR derived phasiRNA (PDPs) and their expression.

626 Supplemental Dataset 5. Comparison of phasiRNA derived from 23 A. thaliana PPRs and the 627 PDPs along with their read counts and sequence.

628 Supplemental Dataset 6. AGO1 and AGO4 associated PDPs.

629 Supplemental Dataset 7. Significant small RNA cluster (p value <0.01) on pseudo- $P P R$. Table 630 also includes the phasiRNA and their expression in different plants identified from highly 631 significant cluster (Start 746-End 997).

632 Supplemental Dataset 8. List of identified targets of PDP 956-977 (+) using PAREsnip tool.

633 Supplemental Dataset 9. Test of significance of variation (ANOVA) for the measured 634 physiological and morphological traits along with pair-wise comparison. 
635 Supplemental Dataset 10. List of primers and their sequences used in the study.

636 Supplemental Dataset 11. Sequencing and mapping read statistics of the small RNA libraries

637 analyzed in the study.

\section{ACKNOWLEDGEMENTS}

639 The authors acknowledge the financial support of the Department of Biotechnology (DBT),

640 (grant \# BT/PR23518/BPA/118/236/2017), Government of India, and partly by Council of 641 Scientific and Industrial Research (CSIR), New Delhi, India. AKV also acknowledge the

642 University Grant Commission (UGC), New Delhi for providing the fellowship. The authors are

643 thankful to in-charge, scanning electron microscope, central instrumentation facility at 644 CSIR_NBRI, Lucknow, UP, India. The institutional ethic committee reference number for the manuscript is CSIR-NBRI_MS/2020/11/03.

\section{AUTHOR CONTRIBUTIONS}

647 AMT conducted experiments, analyzed the data and drafted the MS; RS, AKV and PM 648 conducted experiments; AS analyzed the data and drafted the MS; SN and PAS helped and 649 conducted physiological experiments; SR designed experiments, analyzed data and wrote the 650 MS.

\section{REFERENCES}

652 Adenot, X., Elmayan, T., Lauressergues, D., Boutet, S., Bouché, N., Gasciolli, V., and 653 Vaucheret, H. (2006). DRB4-dependent TAS3 trans-acting siRNAs control leaf morphology 654 through AGO7. Current Biology 16, 927-932.

655 Allen, E., Xie, Z., Gustafson, A.M., and Carrington, J.C. (2005). microRNA-directed phasing 656 during trans-acting siRNA biogenesis in plants. Cell 121, 207-221.

657 Alonso-Blanco, C., Andrade, J., Becker, C., Bemm, F., Bergelson, J., Borgwardt, K.M., Cao, J., 658 Chae, E., Dezwaan, T.M., and Ding, W. (2016). 1,135 genomes reveal the global pattern of polymorphism in Arabidopsis thaliana. Cell 166, 481-491.

660 Alonso-Blanco, C., and Koornneef, M. (2000). Naturally occurring variation in Arabidopsis: an 661 underexploited resource for plant genetics. Trends in plant science 5, 22-29.

662 An, Y., Furber, K.L., and Ji, S. (2017). Pseudogenes regulate parental gene expression via ce 663 RNA network. Journal of cellular and molecular medicine 21, 185-192.

664 Andrés, Z., Pérez-Hormaeche, J., Leidi, E.O., Schlücking, K., Steinhorst, L., McLachlan, D.H., 665 Schumacher, K., Hetherington, A.M., Kudla, J., and Cubero, B. (2014). Control of vacuolar 666 dynamics and regulation of stomatal aperture by tonoplast potassium uptake. Proceedings of the 667 National Academy of Sciences 111, E1806-E1814. 
Axtell, M.J., Jan, C., Rajagopalan, R., and Bartel, D.P. (2006). A two-hit trigger for siRNA biogenesis in plants. Cell 127, 565-577.

670 Barragán, V., Leidi, E.O., Andrés, Z., Rubio, L., De Luca, A., Fernández, J.A., Cubero, B., and

671 Pardo, J.M. (2012). Ion exchangers NHX1 and NHX2 mediate active potassium uptake into vacuoles to regulate cell turgor and stomatal function in Arabidopsis. The Plant Cell 24, 11271142.

674 Barrs, H., and Weatherley, P. (1962). A re-examination of the relative turgidity technique for estimating water deficits in leaves. Australian journal of biological sciences 15, 413-428. Bartel, D.P. (2004). MicroRNAs: genomics, biogenesis, mechanism, and function. cell 116, 281297.

Bassil, E., Tajima, H., Liang, Y.-C., Ohto, M.-a., Ushijima, K., Nakano, R., Esumi, T., Coku, A., Belmonte, M., and Blumwald, E. (2011). The Arabidopsis Na+/H+ antiporters NHX1 and NHX2 control vacuolar $\mathrm{pH}$ and $\mathrm{K}+$ homeostasis to regulate growth, flower development, and reproduction. The Plant Cell 23, 3482-3497. mechanism underlies the biogenesis of plant microRNAs miR319 and miR159. The EMBO journal 28, 3646-3656. Bologna, N.G., Schapire, A.L., Zhai, J., Chorostecki, U., Boisbouvier, J., Meyers, B.C., and Palatnik, J.F. (2013). Multiple RNA recognition patterns during microRNA biogenesis in plants. Genome research 23, 1675-1689.

Bomblies, K., Shukla, V., and Graham, C. (2008). Scanning Electron Microscopy (SEM) of Plant Tissues. CSH protocols 2008, pdb. prot4933. Görlach, J. (2001). Growth stage-based phenotypic analysis of Arabidopsis: a model for high throughput functional genomics in plants. The Plant Cell 13, 1499-1510. validation of a microRNA-directed tandem trans-acting siRNA cascade in Arabidopsis. Proceedings of the National Academy of Sciences 104, 3318-3323.

698 Cuperus, J.T., Carbonell, A., Fahlgren, N., Garcia-Ruiz, H., Burke, R.T., Takeda, A., Sullivan, C.M., Gilbert, S.D., Montgomery, T.A., and Carrington, J.C. (2010). Unique functionality of 22nt miRNAs in triggering RDR6-dependent siRNA biogenesis from target transcripts in Arabidopsis. Nature structural \& molecular biology 17, 997. wide identification and testing of superior reference genes for transcript normalization in Arabidopsis. Plant physiology 139, 5-17.

Dai, X., and Zhao, P.X. (2011). psRNATarget: a plant small RNA target analysis server. Nucleic acids research 39 , W155-W159. miR390 targeting event is sufficient for triggering TAS3-tasiRNA biogenesis in Arabidopsis. Nucleic acids research 45, 5539-5554. cosuppression in petunia. Rna 15, 1965-1970. 
Deng, P., Muhammad, S., Cao, M., and Wu, L. (2018). Biogenesis and regulatory hierarchy of phased small interfering RNAs in plants. Plant biotechnology journal 16, 965-975. Fahlgren, N., Howell, M.D., Kasschau, K.D., Chapman, E.J., Sullivan, C.M., Cumbie, J.S., Givan, S.A., Law, T.F., Grant, S.R., and Dangl, J.L. (2007). High-throughput sequencing of Arabidopsis microRNAs: evidence for frequent birth and death of MIRNA genes. PloS one 2, e219.

Fahlgren, N., Jogdeo, S., Kasschau, K.D., Sullivan, C.M., Chapman, E.J., Laubinger, S., Smith, L.M., Dasenko, M., Givan, S.A., and Weigel, D. (2010). MicroRNA gene evolution in Arabidopsis lyrata and Arabidopsis thaliana. The Plant Cell 22, 1074-1089.

Fei, Q., Xia, R., and Meyers, B.C. (2013). Phased, secondary, small interfering RNAs in posttranscriptional regulatory networks. The Plant Cell 25, 2400-2415.

Folkes, L., Moxon, S., Woolfenden, H.C., Stocks, M.B., Szittya, G., Dalmay, T., and Moulton, V. (2012). PAREsnip: a tool for rapid genome-wide discovery of small RNA/target interactions evidenced through degradome sequencing. Nucleic acids research 40, e103-e103.

Guo, X., Zhang, Z., Gerstein, M.B., and Zheng, D. (2009). Small RNAs originated from pseudogenes: cis-or trans-acting? PLoS computational biology 5, e1000449.

Hirsch, J., Lefort, V., Vankersschaver, M., Boualem, A., Lucas, A., Thermes, C., d'AubentonCarafa, Y., and Crespi, M. (2006). Characterization of 43 non-protein-coding mRNA genes in Arabidopsis, including the MIR162a-derived transcripts. Plant physiology 140, 1192-1204.

Howell, M.D., Fahlgren, N., Chapman, E.J., Cumbie, J.S., Sullivan, C.M., Givan, S.A., Kasschau, K.D., and Carrington, J.C. (2007). Genome-wide analysis of the RNA-DEPENDENT RNA POLYMERASE6/DICER-LIKE4 pathway in Arabidopsis reveals dependency on miRNAand tasiRNA-directed targeting. The Plant Cell 19, 926-942.

Huang, J., Wang, R., Dai, X., Feng, J., Zhang, H., and Zhao, P.X. (2019). A microRNA biogenesis-like pathway for producing phased small interfering RNA from a long non-coding RNA in rice. Journal of experimental botany 70, 1767-1774. Jia, J., Ji, R., Li, Z., Yu, Y., Nakano, M., Long, Y., Feng, L., Qin, C., Lu, D., and Zhan, J. (2020). Soybean DICER-LIKE2 Regulates Seed Coat Color via Production of Primary 22Nucleotide Small Interfering RNAs from Long Inverted Repeats. The Plant Cell 32, 3662-3673. Kurihara, Y., and Watanabe, Y. (2004). Arabidopsis micro-RNA biogenesis through Dicer-like 1 protein functions. Proceedings of the National Academy of Sciences 101, 12753-12758.

Langmead, B., and Salzberg, S.L. (2012). Fast gapped-read alignment with Bowtie 2. Nature methods 9, 357.

Liu, Y., Teng, C., Xia, R., and Meyers, B.C. (2020). PhasiRNAs in Plants: Their Biogenesis, Genic Sources, and Roles in Stress Responses, Development, and Reproduction. The Plant Cell 32, 3059-3080. Lurin, C., Andrés, C., Aubourg, S., Bellaoui, M., Bitton, F., Bruyère, C., Caboche, M., Debast, C., Gualberto, J., and Hoffmann, B. (2004). Genome-wide analysis of Arabidopsis pentatricopeptide repeat proteins reveals their essential role in organelle biogenesis. The Plant

753 Ma, Z., Jiang, J., Hu, Z., Lyu, T., Yang, Y., Jiang, J., and Cao, J. (2017). Over-expression of 754 miR158 causes pollen abortion in Brassica campestris ssp. chinensis. Plant molecular biology 93, 755 313-326.

756 Marin, E., Jouannet, V., Herz, A., Lokerse, A.S., Weijers, D., Vaucheret, H., Nussaume, L., 757 Crespi, M.D., and Maizel, A. (2010). miR390, Arabidopsis TAS3 tasiRNAs, and their AUXIN 
RESPONSE FACTOR targets define an autoregulatory network quantitatively regulating lateral root growth. The Plant Cell 22, 1104-1117.

760 Mateos, J.L., Bologna, N.G., Chorostecki, U., and Palatnik, J.F. (2010). Identification of microRNA processing determinants by random mutagenesis of Arabidopsis MIR172a precursor. Current Biology 20, 49-54. May, P., Liao, W., Wu, Y., Shuai, B., McCombie, W.R., Zhang, M.Q., and Liu, Q.A. (2013). The effects of carbon dioxide and temperature on microRNA expression in Arabidopsis development. Nature communications 4. Mitchell-Olds, T., and Schmitt, J. (2006). Genetic mechanisms and evolutionary significance of natural variation in Arabidopsis. Nature 441, 947-952.

Ng, D.W., Zhang, C., Miller, M., Palmer, G., Whiteley, M., Tholl, D., and Chen, Z.J. (2011). cisand trans-Regulation of miR163 and target genes confers natural variation of secondary metabolites in two Arabidopsis species and their allopolyploids. The Plant Cell 23, 1729-1740.

Rajagopalan, R., Vaucheret, H., Trejo, J., and Bartel, D.P. (2006). A diverse and evolutionarily fluid set of microRNAs in Arabidopsis thaliana. Genes \& development 20, 3407-3425.

Reinhart, B.J., Weinstein, E.G., Rhoades, M.W., Bartel, B., and Bartel, D.P. (2002). MicroRNAs in plants. Genes \& development 16, 1616-1626. Ryan, B.M., Robles, A.I., and Harris, C.C. (2010). Genetic variation in microRNA networks: the implications for cancer research. Nature Reviews Cancer 10, 389-402.

Schmittgen, T.D., and Livak, K.J. (2008). Analyzing real-time PCR data by the comparative CT method. Nature protocols 3, 1101-1108. Schmitz-Linneweber, C., and Small, I. (2008). Pentatricopeptide repeat proteins: a socket set for organelle gene expression. Trends in plant science 13, 663-670.

Shahid, S., Kim, G., Johnson, N.R., Wafula, E., Wang, F., Coruh, C., Bernal-Galeano, V., Phifer, Cuscuta campestris target host messenger RNAs. Nature 553, 82-85.

Sosa-Valencia, G., Palomar, M., Covarrubias, A.A., and Reyes, J.L. (2017). The legume miR1514a modulates a NAC transcription factor transcript to trigger phasiRNA formation in response to drought. Journal of experimental botany 68, 2013-2026. Moulton, V. (2018). The UEA sRNA Workbench (version 4.4): a comprehensive suite of tools for analyzing miRNAs and sRNAs. Bioinformatics 34, 3382-3384. Arabidopsis thaliana HYL1-dependent pri-miRNAs. Nucleic acids research 37, 3083-3093. Tam, O.H., Aravin, A.A., Stein, P., Girard, A., Murchison, E.P., Cheloufi, S., Hodges, E., Anger, M., Sachidanandam, R., and Schultz, R.M. (2008). Pseudogene-derived small interfering RNAs regulate gene expression in mouse oocytes. Nature 453, 534-538. evolutionary genetics analysis version 6.0. Molecular biology and evolution 30, 2725-2729.

798 Thatcher, S.R., Burd, S., Wright, C., Lers, A., and Green, P.J. (2015). Differential expression of miRNAs and their target genes in senescing leaves and siliques: insights from deep sequencing of small RNAs and cleaved target RNAs. Plant, cell \& environment 38, 188-200.

802

Thibaud-Nissen, F., Ouyang, S., and Buell, C.R. (2009). Identification and characterization of pseudogenes in the rice gene complement. BMC genomics 10, 317. 
Todesco, M., Balasubramanian, S., Cao, J., Ott, F., Sureshkumar, S., Schneeberger, K., Meyer, R.C., Altmann, T., and Weigel, D. (2012). Natural variation in biogenesis efficiency of individual Arabidopsis thaliana microRNAs. Current Biology 22, 166-170. Tripathi, A.M., Singh, A., Singh, R., Verma, A.K., and Roy, S. (2019). Modulation of miRNA expression in natural populations of A. thaliana along a wide altitudinal gradient of Indian Himalayas. Scientific reports 9, 1-16.

Tyagi, A., Yadav, A., Tripathi, A.M., and Roy, S. (2016). High light intensity plays a major role in emergence of population level variation in Arabidopsis thaliana along an altitudinal gradient. Scientific reports $6,26160$. Varkonyi-Gasic, E., Wu, R., Wood, M., Walton, E.F., and Hellens, R.P. (2007). Protocol: a highly sensitive RT-PCR method for detection and quantification of microRNAs. Plant methods 3, 1-12. Vazquez, F., Vaucheret, H., Rajagopalan, R., Lepers, C., Gasciolli, V., Mallory, A.C., Hilbert, J.L., Bartel, D.P., and Crété, P. (2004). Endogenous trans-acting siRNAs regulate the accumulation of Arabidopsis mRNAs. Molecular cell 16, 69-79.

818 Voinnet, O. (2009). Origin, biogenesis, and activity of plant microRNAs. Cell 136, 669-687.

819 Wang, H., Zhang, X., Liu, J., Kiba, T., Woo, J., Ojo, T., Hafner, M., Tuschl, T., Chua, N.H., and Wang, X.J. (2011). Deep sequencing of small RNAs specifically associated with Arabidopsis AGO1 and AGO4 uncovers new AGO functions. THe plant journal 67, 292-304.

Wang, J., Yang, X., Xu, H., Chi, X., Zhang, M., and Hou, X. (2012). Identification and characterization of microRNAs and their target genes in Brassica oleracea. Gene 505, 300-308. Wang, Y., Liu, W., Shen, H., Zhu, X., Zhai, L., Xu, L., Wang, R., Gong, Y., Limera, C., and Liu, L. (2015). Identification of radish (Raphanus sativus L.) miRNAs and their target genes to explore miRNA-mediated regulatory networks in lead $(\mathrm{Pb})$ stress responses by high-throughput sequencing and degradome analysis. Plant Molecular Biology Reporter 33, 358-376.

Watanabe, T., Totoki, Y., Toyoda, A., Kaneda, M., Kuramochi-Miyagawa, S., Obata, Y., Chiba, H., Kohara, Y., Kono, T., and Nakano, T. (2008). Endogenous siRNAs from naturally formed dsRNAs regulate transcripts in mouse oocytes. Nature 453, 539-543.

Werner, S., Wollmann, H., Schneeberger, K., and Weigel, D. (2010). Structure determinants for accurate processing of miR172a in Arabidopsis thaliana. Current Biology 20, 42-48.

Williams, L., Carles, C.C., Osmont, K.S., and Fletcher, J.C. (2005). A database analysis method identifies an endogenous trans-acting short-interfering RNA that targets the Arabidopsis ARF2, ARF3, and ARF4 genes. Proceedings of the National Academy of Sciences 102, 9703-9708.

Xia, R., Meyers, B.C., Liu, Z., Beers, E.P., Ye, S., and Liu, Z. (2013). MicroRNA superfamilies descended from miR390 and their roles in secondary small interfering RNA biogenesis in eudicots. The Plant Cell 25, 1555-1572. Xia, R., Xu, J., and Meyers, B.C. (2017). The emergence, evolution, and diversification of the miR390-TAS3-ARF pathway in land plants. The Plant Cell 29, 1232-1247.

Xia, R., Ye, S., Liu, Z., Meyers, B.C., and Liu, Z. (2015). Novel and recently evolved microRNA clusters regulate expansive F-BOX gene networks through phased small interfering RNAs in wild diploid strawberry. Plant physiology 169, 594-610.

844 Xia, R., Zhu, H., An, Y.-q., Beers, E.P., and Liu, Z. (2012). Apple miRNAs and tasiRNAs with novel regulatory networks. Genome biology 13, R47. Expression of Arabidopsis MIRNA genes. Plant physiology 138, 2145-2154. 
Xu, M.Y., Dong, Y., Zhang, Q.X., Zhang, L., Luo, Y.Z., Sun, J., Fan, Y.L., and Wang, L. (2012). Identification of miRNAs and their targets from Brassica napus by high-throughput sequencing and degradome analysis. BMC genomics 13, 421. Yagi, Y., Tachikawa, M., Noguchi, H., Satoh, S., Obokata, J., and Nakamura, T. (2013). Pentatricopeptide repeat proteins involved in plant organellar RNA editing. RNA biology 10, 1419-1425.

854 Yoshikawa, M., Peragine, A., Park, M.Y., and Poethig, R.S. (2005). A pathway for the biogenesis of trans-acting siRNAs in Arabidopsis. Genes \& development 19, 2164-2175. Yu, G., Yao, W., Gumireddy, K., Li, A., Wang, J., Xiao, W., Chen, K., Xiao, H., Li, H., and Tang, K. (2014). Pseudogene PTENP1 functions as a competing endogenous RNA to suppress clear-cell renal cell carcinoma progression. Molecular cancer therapeutics 13, 3086-3097. Zhai, J., Jeong, D.-H., De Paoli, E., Park, S., Rosen, B.D., Li, Y., González, A.J., Yan, Z., Kitto, S.L., and Grusak, M.A. (2011). MicroRNAs as master regulators of the plant NB-LRR defense gene family via the production of phased, trans-acting siRNAs. Genes \& development 25, 25402553. regulatory pathways. Bioinformatics 30, 1045-1046. Zhang, Z., Guo, X., Ge, C., Ma, Z., Jiang, M., Li, T., Koiwa, H., Yang, S.W., and Zhang, X. (2017). KETCH1 imports HYL1 to nucleus for miRNA biogenesis in Arabidopsis. Proceedings of the National Academy of Sciences 114, 4011-4016. Zhong, S.-H., Liu, J.-Z., Jin, H., Lin, L., Li, Q., Chen, Y., Yuan, Y.-X., Wang, Z.-Y., Huang, H., and Qi, Y.-J. (2013). Warm temperatures induce transgenerational epigenetic release of RNA silencing by inhibiting siRNA biogenesis in Arabidopsis. Proceedings of the National Academy of Sciences 110, 9171-9176. Tadege, M. (2013). The trans-acting short interfering RNA3 pathway and no apical meristem antagonistically regulate leaf margin development and lateral organ separation, as revealed by analysis of an argonaute7/lobed leaflet1 mutant in Medicago truncatula. The Plant Cell 25, 48454862. 
Tables

Table 1. Comparative read counts of PDPs and related small RNAs in different populations

\begin{tabular}{|c|c|c|c|c|c|c|c|c|c|c|c|c|}
\hline & & \multicolumn{3}{|l|}{ Deh } & \multicolumn{2}{|l|}{ Mun } & \multicolumn{3}{|c|}{ Chit } & \multicolumn{3}{|c|}{ Col-0 } \\
\hline & & $\begin{array}{c}\text { Replicate } \\
1 \\
\end{array}$ & \multicolumn{2}{|c|}{$\begin{array}{c}\text { Replicate } \\
2 \\
\end{array}$} & $\begin{array}{c}\text { Replicate } \\
1 \\
\end{array}$ & $\begin{array}{c}\text { Replicate } \\
2 \\
\end{array}$ & \multicolumn{2}{|c|}{$\begin{array}{c}\text { Replicate } \\
1 \\
\end{array}$} & $\begin{array}{c}\text { Replicate } \\
2 \\
\end{array}$ & \multicolumn{2}{|c|}{$\begin{array}{c}\text { Replicate } \\
1 \\
\end{array}$} & $\begin{array}{c}\text { Replicate } \\
2 \\
\end{array}$ \\
\hline \multicolumn{2}{|c|}{ miR166e-3p } & 15934 & \multicolumn{2}{|l|}{11399} & 10459 & 10991 & \multicolumn{2}{|c|}{11356} & 12860 & \multicolumn{2}{|c|}{15400} & 7339 \\
\hline \multicolumn{2}{|c|}{ miR158 } & 60210 & \multicolumn{2}{|l|}{17443} & 3848 & 4707 & \multicolumn{2}{|c|}{23242} & 27121 & \multicolumn{2}{|c|}{70440} & 37725 \\
\hline \multicolumn{2}{|l|}{ siR9 } & 595 & \multicolumn{2}{|l|}{792} & 614 & 743 & \multicolumn{2}{|c|}{337} & 391 & 740 & & 428 \\
\hline siR12 & & 887 & 221 & & 548 & 579 & 480 & & 412 & 664 & & 397 \\
\hline $\begin{array}{l}\text { PDPs } \\
\text { expression }\end{array}$ & & 172 & 105 & & 279 & 288 & 60 & & 75 & 107 & & 62 \\
\hline & Col- & -0 (NTL) & $\begin{array}{r}\text { miR15 } \\
\text { n }\end{array}$ & $\begin{array}{l}\text { 8muta } \\
t\end{array}$ & $\begin{array}{r}\text { Pse } \\
\text { PPR }\end{array}$ & $\begin{array}{l}\text { do- } \\
\text { utant }\end{array}$ & DTL & & IL & Col-0 & $\begin{array}{c}\text { miR15 } \\
\text { 8OE }\end{array}$ & \begin{tabular}{|c} 
Pseudo \\
- \\
PPRO \\
E
\end{tabular} \\
\hline & $\begin{array}{l}\text { Replica } \\
\text { te } 1\end{array}$ & $\begin{array}{l}\text { Replica } \\
\text { te } 2\end{array}$ & $\begin{array}{l}\text { Repli } \\
\text { cate } 1\end{array}$ & $\begin{array}{l}\text { Repl } \\
\text { cate }\end{array}$ & $\begin{array}{l}\text { Replica } \\
\text { te } 1\end{array}$ & $\begin{array}{l}\text { Replica } \\
\text { te } 2\end{array}$ & $\begin{array}{l}\text { Repli } \\
\text { cate } 1\end{array}$ & $\begin{array}{l}\text { Repli } \\
\text { cate } 1\end{array}$ & $\begin{array}{l}\text { Replica } \\
\text { te } 2\end{array}$ & $\begin{array}{l}\text { Replica } \\
\text { te } 1\end{array}$ & $\begin{array}{l}\text { Replica } \\
\text { te } 1\end{array}$ & $\begin{array}{c}\text { Replica } \\
\text { te } 1\end{array}$ \\
\hline $\begin{array}{c}\text { mir166e- } \\
3 p \\
\end{array}$ & 16026 & 17135 & 17941 & 18588 & 16318 & 17456 & 18355 & 23821 & 14525 & 54950 & 35107 & 41774 \\
\hline $\begin{array}{c}\text { miR158a- } \\
3 p \\
\end{array}$ & 224638 & 164825 & 3208 & 2853 & 230162 & 344228 & 687 & 25228 & 42200 & 308014 & 2991449 & 389142 \\
\hline siR9 & 1573 & 1231 & 1789 & 2231 & 1691 & 2679 & 1975 & 1083 & 2210 & 3061 & 2256 & 2466 \\
\hline $\mathrm{siR} 12$ & 734 & 258 & 1225 & 833 & 470 & 1125 & 609 & 600 & 414 & 991 & 1016 & 1025 \\
\hline $\begin{array}{c}\text { PDPs } \\
\text { expression }\end{array}$ & 174 & 184 & 241 & 265 & 113 & 129 & 552 & 419 & 386 & 228 & 169 & 1641 \\
\hline
\end{tabular}

890 Expression (read counts) of miR158, tasiRNAs and PDPs in different west Himalayan populations and those of Col-0, SALK mutants and over expression lines along with DTL and ITL are shown in above and lower panel, respectively. The expression of miR166 was considered as technical standard being not associated with PDPs and related siRNAs pathway. 
A

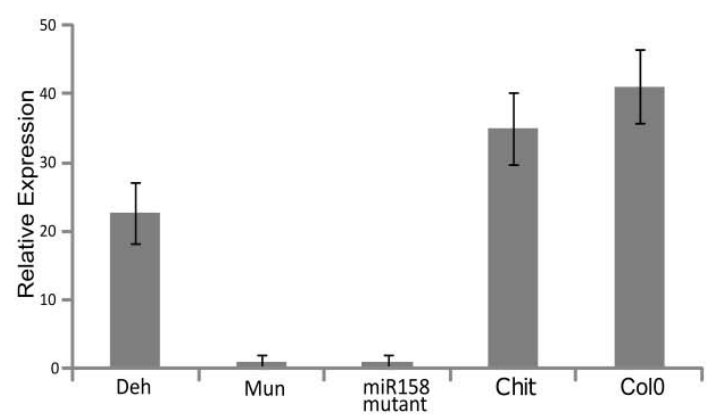

C
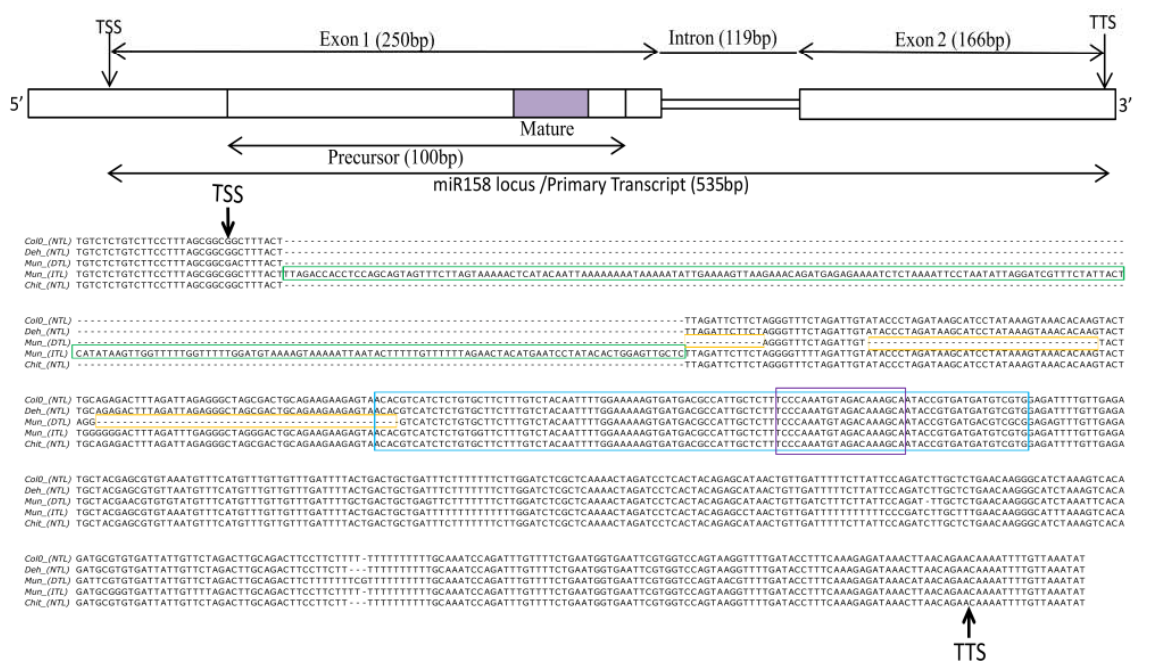

B

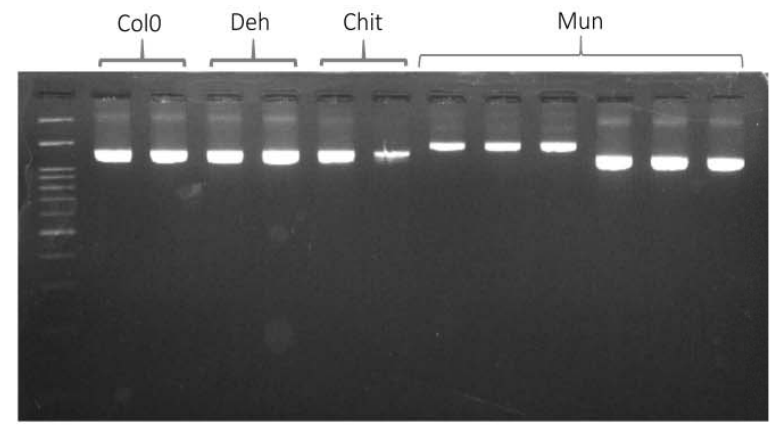

D

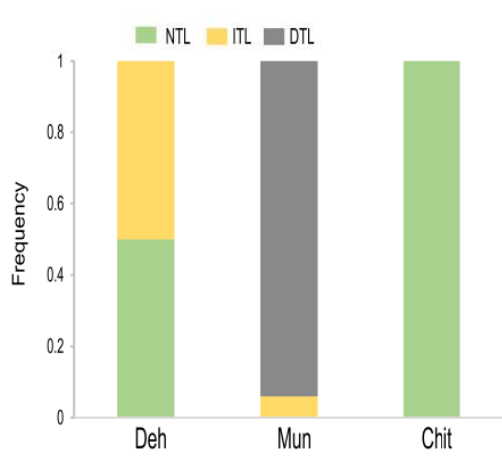

905

906

907

908

909

910

911

912

913

914

915

916

917

918

919

920

921

922

\section{Figure 1: Expression pattern and length polymorphisms of miR158 locus in different populations}

(A) The expression pattern of miR158 in different west Himalayan populations as well as miR158 SALK mutant and Col-0 were analyzed by qRT-PCR. The data represents the mean of four independent biological replicates \pm SD.

(B) Length polymorphism of miR158 locus as revealed by agarose gel electrophoresis of the PCR amplification products from a few individuals of each of the three populations. While Deh, Chit and Col-0 exhibited same fragment length as expected, the Mun exhibited two types of fragment lengths due to insertion and deletion.

(C) Multiple alignment of miR158 locus sequenced from the three populations. The insertion sequence in ITL, insertion type locus and the deleted sequence in DTL, deleted type locus are shown in green and yellow box, respectively. The precursor and mature sequences of miR158 are shown in blue and violet boxes, respectively. TSS, transcription start site and TTS, transcription termination site are indicated by arrows. The locus was annotated against Col-0 reference locus and schematically represented above the aligned sequences.

(D) Frequency of insertion/deletion in miR158 locus in west Himalayan Arabidopsis populations as analyzed from 50 plants of from each population. 

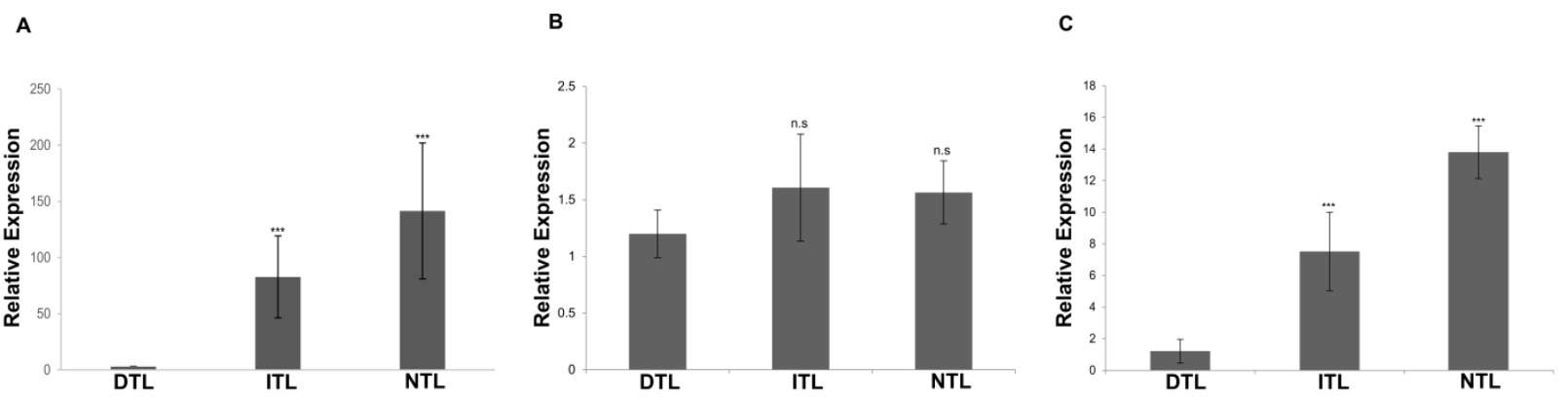

D

E

$\mathrm{F}$

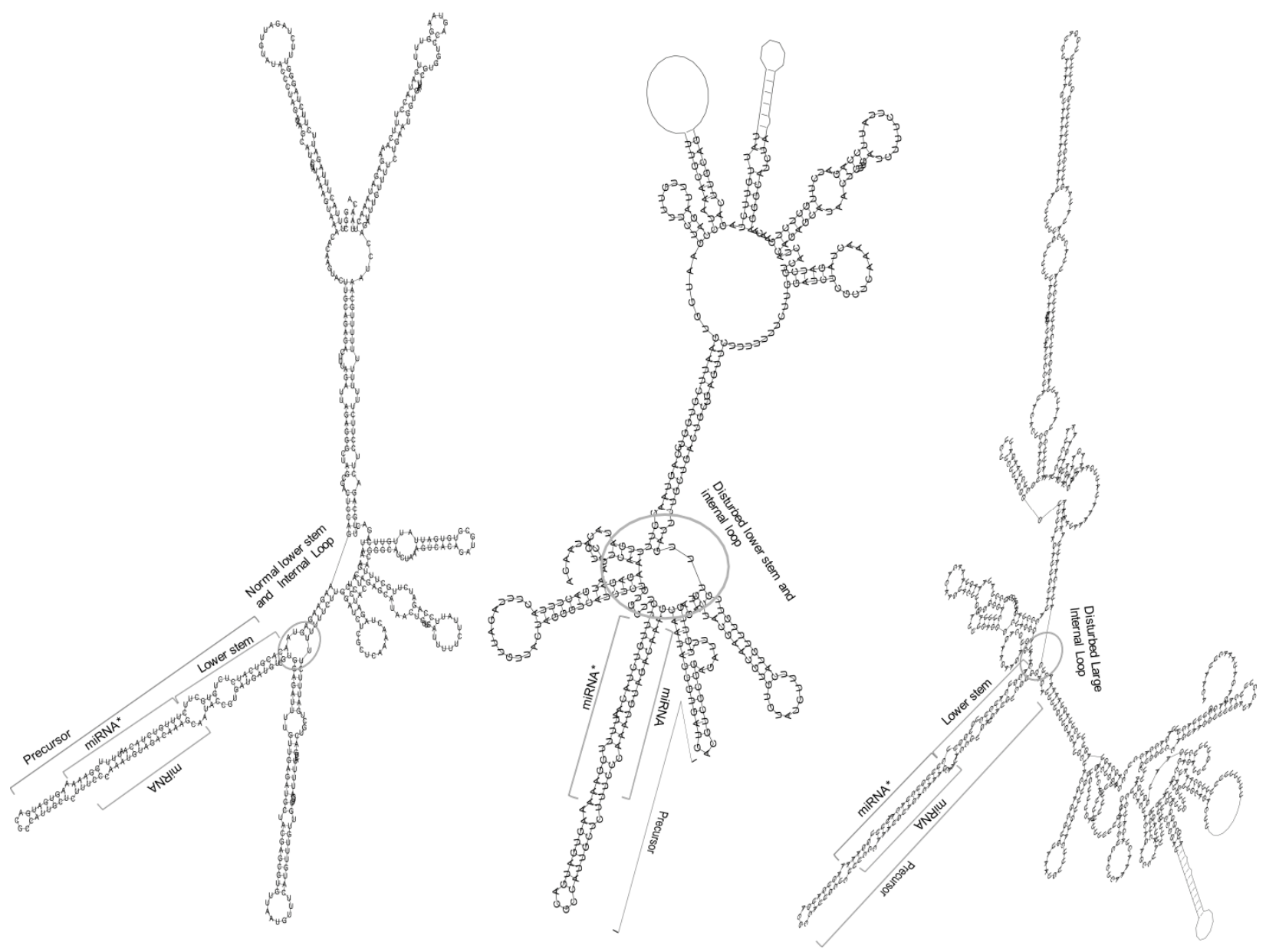

924 Fig. 2: Expression patterns of miR158, pri-miR158, pre-miR158 and predicted secondary structures of pri-miR158

Relative expression pattern of: (A) miR158; (B) pri-miR158; and (C) pre-miR158, in DTL, ITL and NTL plants. The data represents the mean of three independent biological replicates $\pm \mathrm{SD}$. $\left(* * * \mathrm{P}<0.001,{ }^{* *} \mathrm{P} \leq 0.01 . * \mathrm{P} \leq 0.05\right.$, (Student's t-test); ns, nonsignificant). derived from the respective sequence using RNAfold web server. The miRNA/miRNA*, precursor, lower stem sequences are shown along with disturbed large internal loops in DTL and ITL (encircled). 
A

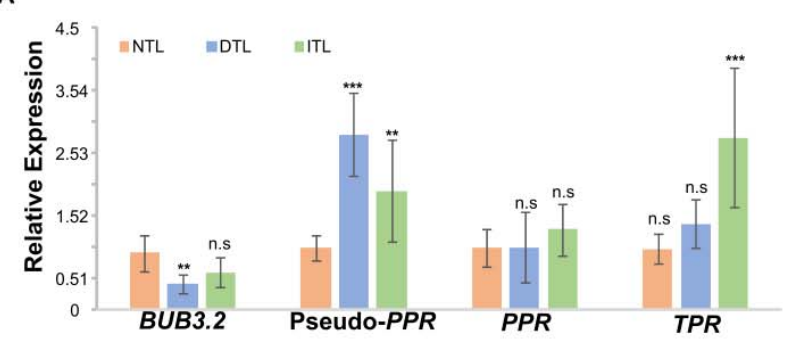

C

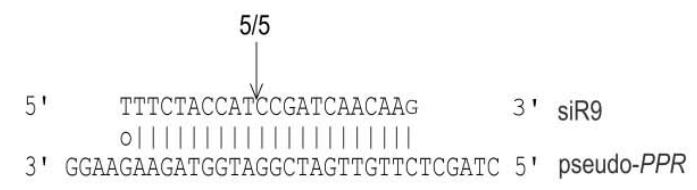

B

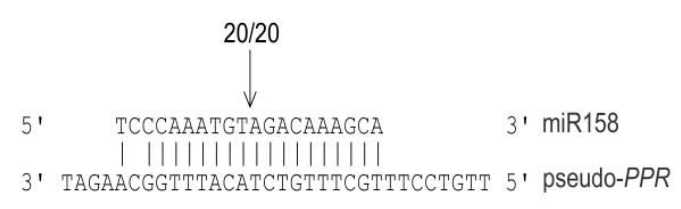

D

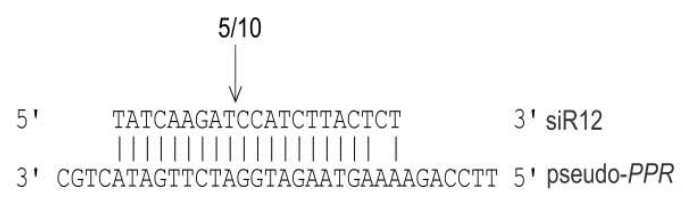

\section{Fig. 3: miR158 target expression pattern and target validation of miR158 and tasiRNA}

936

937

938

939

940

941

942
(A) The expression pattern of all the predicted targets of miR158 as evaluated by qRT-PCR. The data represents the mean of three independent biological replicates \pm SD $(* * * \mathrm{P}<0.001, * * \mathrm{P} \leq 0.01$. * $\mathrm{P} \leq 0.05$, (Student's t-test), ns (not significant)). Pseudo- $P P R$ as the target of (B) miR158; (C) siR9; and (D) siR12, validated using modified 5' RLM-RACE. Pseudo-PPRmRNA:miRNA/tasiRNA alignments are shown along with the detected cleavage site (shown by arrow). The number above arrow indicates the positive clones at this cleavage site/total number of colonies sequenced. 


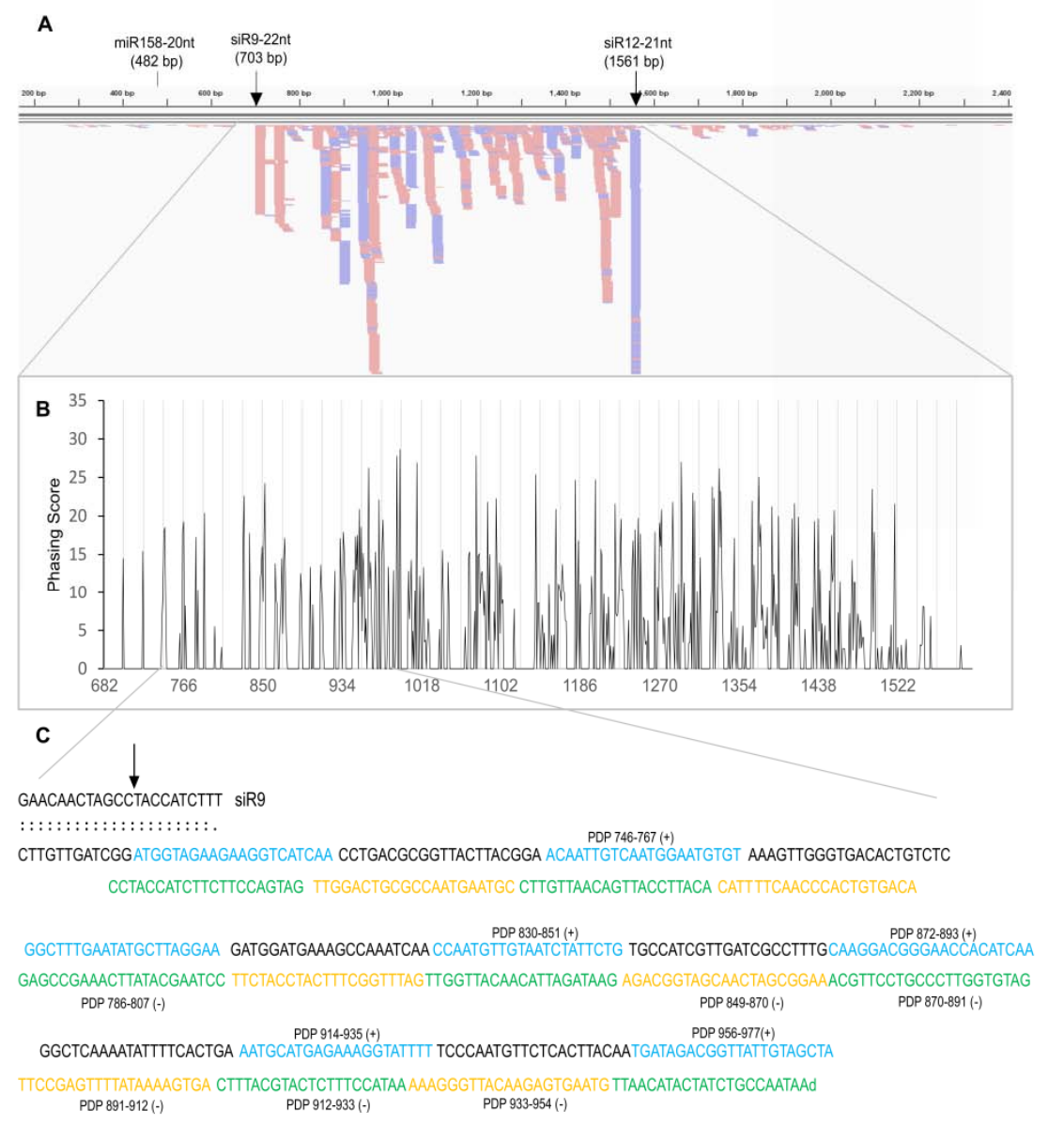

D

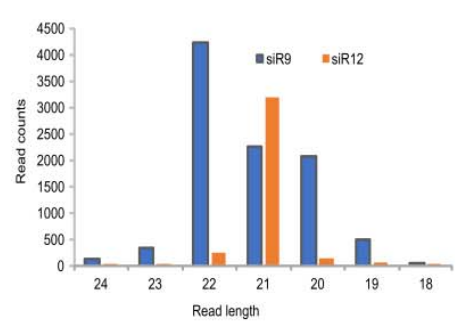

E

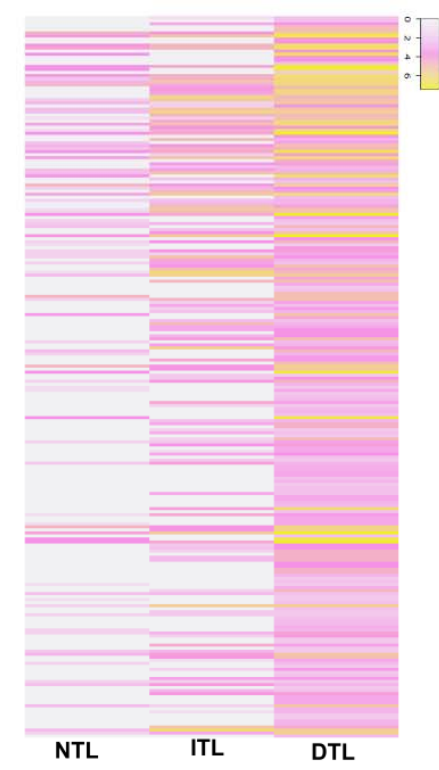

944 Fig. 4: PhasiRNA analysis and expression pattern

(A) The small RNAs were mapped against pseudo- $P P R$ shown as horizontal line along with nucleotide positions and cleavage sites (arrow) of miR158, siR9 and siR12. The red blocks depict reads aligned in forward orientation and blue in reverse orientation (visualized via Integrative Genomics Viewer (IGV)).

(B) The phasing scores calculated using small RNAs mapped between 600-1600 nucleotide positions of pseudo- $P P R$. The $\mathrm{X}$-axis and $\mathrm{Y}$-axis represent the phasing score and nucleotide positions of pseudo- $P P R$, respectively. The 21 nucleotides phasing intervals are shown by vertical grey lines.

(C) The phasing pattern of a significant cluster of phasiRNA derived from pseudo- $P P R$ (PDP) initiated at siR9 cleavage site (marked by arrow). Blue and red sequences indicate the phasiRNAs (PDPs) derived from sense $(+)$ strand while yellow and green sequences represent phasiRNAs derived from antisense (-) strand.

(D) Read length distribution of siR9 and siR12 in Col-0. The data indicates the expression of 22-nucleotide is dominating for siR9.

(E) Heatmap depicting expression pattern of phasiRNAs in NTL, ITL and DTL ( $\mathrm{n}=238$ ). The $\log 2$ value of PDPs read counts are represented by the colour intensity scale. (NTLNormal Type Locus; ITL-Inserted Type Locus; DTL-Deleted Type Locus). 

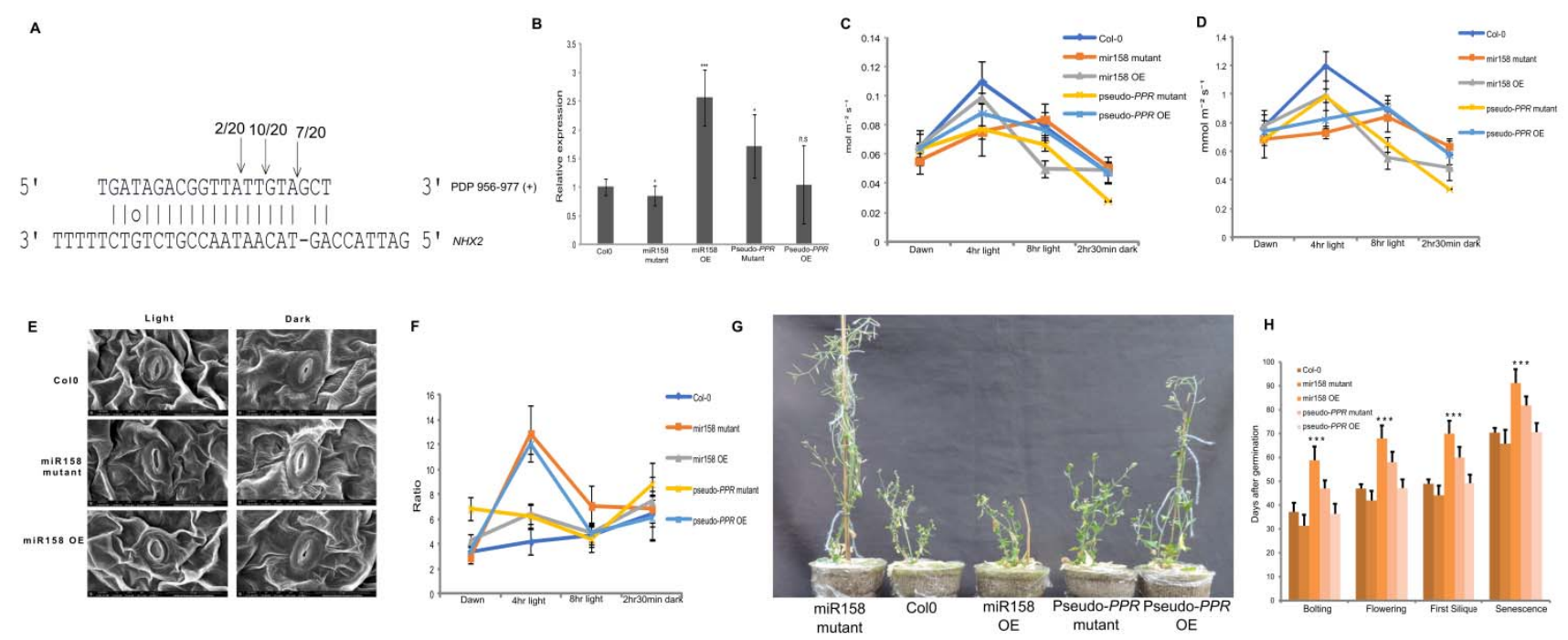

Fig. 5: PDP target analysis and their functional characterizations

(A) The complementary pairing of PDP 956-977(+) with its target $N H X 2$ is shown with arrows indicating the cleaved positions and the numbers suggest positive clones with cleavage sites/total number of colonies sequenced. The target of the highly expressed PDP, PDP 956-977(+) validated using modified 5' RLM-RACE. data represents the mean of three independent biological replicates $\pm \mathrm{SD}$. $(* * * \mathrm{P}<0.001$, $* * \mathrm{P} \leq 0.01$. * $\mathrm{P} \leq 0.05$, (Student's t-test), ns (not significant)).

Physiological parameters of mutant and transgenic lines in Col-0 background measured at different time points: (C) Stomatal conductance; (D) Transpiration rate (E) Scanning Electron microscope image of stomata at two time points (light and $2 \mathrm{hr} 30$ min dark) taken at 8000x (F) Water Use Efficiency. The data represents the mean of five biological replicated \pm standard deviation.

984

(G) Image of plants of miR158 mutant, miR158 OE, pseudo- $P P R$ mutant, pseudo- $P P R$ OE and Col0 taken on 56th day post germination. Plants were grown with normal watering till 21 days and then water was withheld for 35 days after pots were saturated with water on 21 st day.

(H) Measurement of various life cycle related parameters in mutant and transgenic lines in Col-0 background. Life cycle parameters namely, bolting, first flower opening, emergence of first silique and senescence were measured in terms of days after germination. Data represents average of 14-15 plants per genotype \pm SD. Asterisk above graph shows significance of variation ('***' $\mathrm{P}<0.001$, ' $* *$ ' $\mathrm{P}<0.01$, '*' $\mathrm{P}<0.05$, '. $\mathrm{P}<0.1$, ns $=$ not signifiant). 

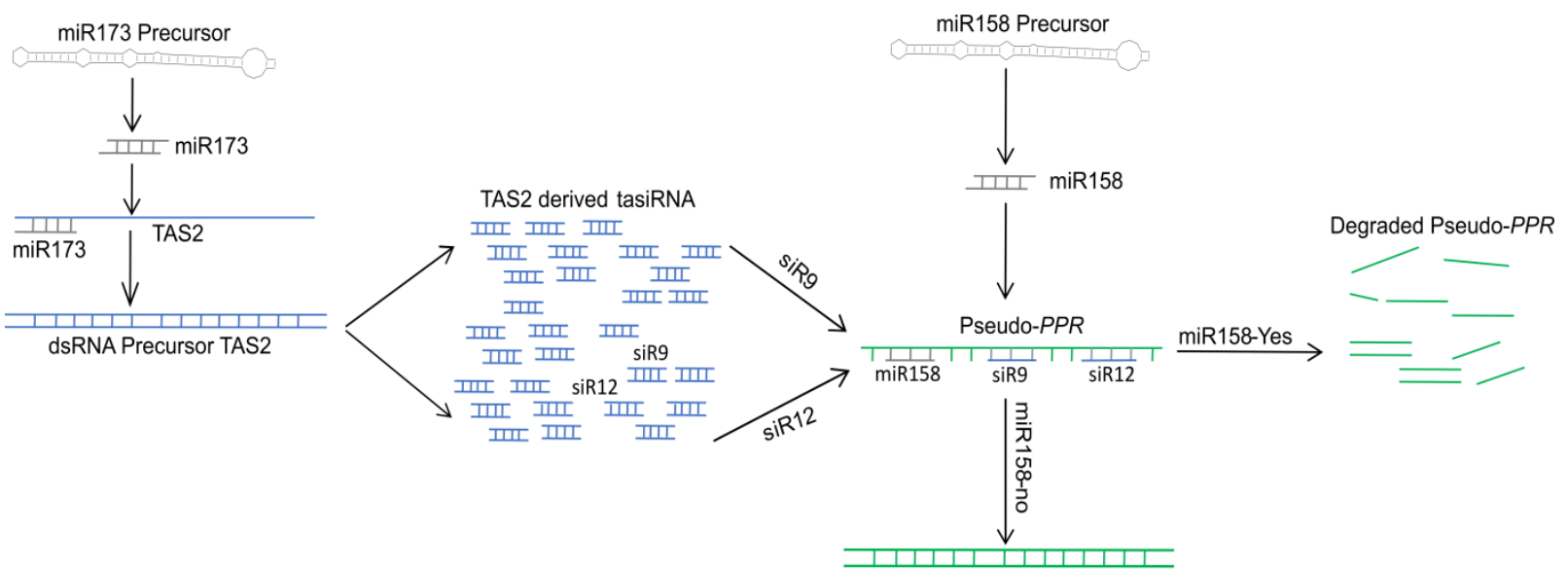

dsRNA Precursor TAS2

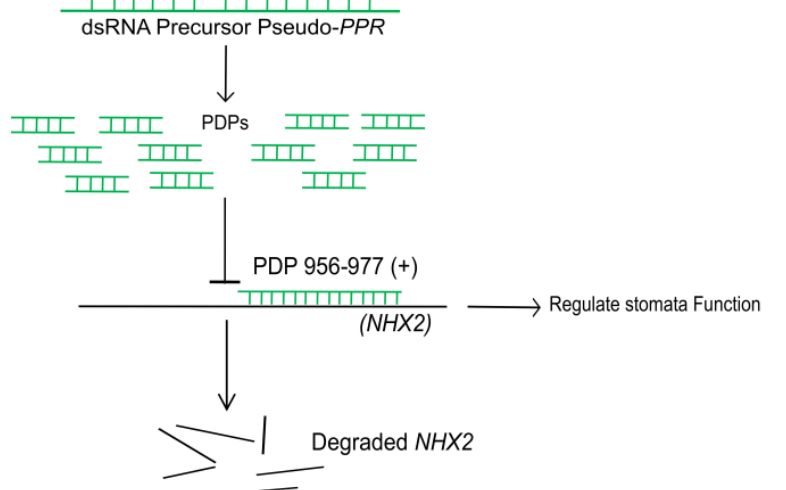

988 Fig. 6: Proposed model for miR158 regulation.

989 The model depicts miR158 acts as a negative regulator of phasiRNA biogenesis from a pseudo$990 P P R$. The pseudo-PPR is targeted by miR 158 besides being targeted by two tasiRNAs, siR9 and 991 siR12 derived from TAS2 locus. While miR158 activity leads to degradation of the pseudo-PPR 992 and thus there is no biogenesis of phasiRNAs, null activity of miR158 makes the pseudo- $P P R$ 993 template available for targeting by the two tasiRAs leading to biogenesis of phasiRNAs. One of 994 the phasiRNA produced by this mechanism targets and degrades the NHX2 gene which in turn 995 regulates stomatal functioning. 